\title{
Upregulation of The Transmembrane Protease Serine 3 (TMPRSS3) mRNA Level In Radioresistant Colorectal Cancer Tissues
}

\section{Jia-Ying Wen}

Guangxi Medical University First Affiliated Hospital

\section{Ye-Ying Fang}

Guangxi Medical University First Affiliated Hospital

\section{Gang Chen}

Guangxi Medical University First Affiliated Hospital

\section{Rong-Quan He}

Guangxi Medical University First Affiliated Hospital

\section{He-Qing Huang}

Guangxi Medical University First Affiliated Hospital

\section{Ren-Sheng Wang}

Guangxi Medical University First Affiliated Hospital

\section{Da-Tong Zeng}

Red Cross Hospital of Yulin City

\section{Wei-Jian Huang}

Red Cross Hospital of Yulin City

Xin-Gan Qin ( $\nabla$ qinxingan_gxmu@163.com )

Guangxi Medical University First Affiliated Hospital https://orcid.org/0000-0002-1232-7030

\section{Research}

Keywords: Colorectal cancer, Transmembrane protease serine 3 (TMPRSS3), Radioresistance, Standardized mean difference (SMD), Area under the curve (AUC), Hazard ratio (HR)

Posted Date: August 16th, 2021

DOl: https://doi.org/10.21203/rs.3.rs-783489/v1

License: (1) (1) This work is licensed under a Creative Commons Attribution 4.0 International License. Read Full License 


\section{Abstract}

\section{Background}

Previous studies have shown that transmembrane protease serine 3 (TMPRSS3) is abnormally expressed in various tumours and its expression is closely related to tumorigenesis and progression [1-3]. However, there have been no reports regarding the role of TMPRSS3 in colorectal cancer (CRC). The aim of the current study was to comprehensively explore the clinical value of TMPRSS3 on the radioresistance of CRC.

\section{Methods}

In this study, we evaluated the expression level of TMPRSS3 in radioresistant CRC tissues as well as CRC tissues by analysing public data sets from the Cancer Genome Atlas (TCGA), Gene Expression Omnibus (GEO), ArrayExpress, and GTEx databases, as well as explored the relationship between TMPRSS3 and lymph node metastasis, distant metastasis, and prognosis. In addition, we investigated the biological function of TMPRSS3 by gene ontology (GO) and analysis of the Kyoto Encyclopaedia of Genes and Genomes (KEGG). Finally, we created a protein-protein interaction (PPI) network to identify hub genes that may function in concert with TMMPRSS3.

\section{Results}

Eight radiation-processed CRC tissue data sets and 26 CRC mRNA data sets were obtained. The pooled analysis revealed that TMPRSS3 was significantly highly expressed in CRC radioresistant tissues $-\mathrm{SMD}=$ 0.38 (95\% Cl: 0.14 0.63; $p$-value < 0.05). In addition, TMPRSS3 is also as highly expressed in CRC tissues $-\mathrm{SMD}=1.55$ (95\% Cl: 1.20 1.90; p-value < 0.05). Subsequently, we explored the relationship between TMPRSS 3 and both metastasis and prognosis. The results revealed no relationship between TMPRSS3 and lymph node metastasis and distant metastasis ( $p$-value $>0.05$ ). Furthermore, pooled analysis of HR revealed that TMPRSS3 could be used as a risk factor for DFS-SMD = 1.28 (95\% Cl: 1.03 1.60; $p$-value < 0.05). Finally, we obtained 429 co-expressed genes for bioinformatics analysis and selected a total of four hub genes: MSLN, MFI2, CKAP4, and PXN. The results revealed that these genes cooperate with TMPRSS3 to participate in biological processes, such as CRC development, metastasis, and radioresistance by affecting autophagy, cell-cell adhesion, and extracellular matrix breakdown.

\section{Conclusion}

This study reveals that TMPRSS3 has potential as a new biomarker and radioresistant therapeutic target for CRC and also has a few implications for the prognosis of CRC patients. However, this conclusion must be further experimentally verified.

\section{Background}


Colorectal carcinoma (CRC) is one of the most common malignant tumours of the digestive system. According to the latest statistical data, the global incidence of CRC is the third highest in malignant tumours and the second highest in terms of mortality in 2020, with 1.88 million new cases and 910,000 deaths in the entire year [4]. CRC seriously endangers human health, thereby leading to the promotion of early screening for CRC and the development of treatment methods in recent years $[5,6]$. In particular, radiotherapy plays an increasingly important role in the treatment of malignant tumours as a local treatment [7-9]. According to 2019 statistics from the American Cancer Society and National Cancer Institute, it is now recognized that surgery is the treatment of choice for patients with stage I rectal cancer, but half of the patients with stage I rectal cancer still require adjuvant chemoradiotherapy [10]; for those with stage II and III rectal cancer, neoadjuvant chemoradiotherapy combined with surgery is usually used [10-13]. However, due to the heterogeneity of tumours and varied sensitivity of tumour cells to radiotherapy, a few patients develop radiotherapy resistance that ultimately leads to tumour progression [14-17]. In addition, it has been shown that approximately $25 \%$ of CRC patients developed colorectal liver cancer metastasis at initial diagnosis, and $20 \%$ of CRC patients experience a recurrence of metastasis after primary tumour resection [18-22]. Currently, the clinical treatment for this group of metastatic CRC patients is limited and the therapeutic effect is poor. The five-year overall survival rate of CRC patients with liver metastases is less than $10 \%$, and the survival time of CRC patients even after surgical resection of metastatic lesions is only 35 months $[23,24]$. Therefore, it is of great significance to study the mechanism of radiotherapy resistance and identify new targets for the treatment of CRC.

Type II transmembrane serine proteases (TTSPS) are a family of cell surface proteases that play a key role in many types of cancer [25]. During the development and progression of cancers, TTSPs play a significant role in tissue remodelling and tumour progression through a series of proteolytic activities [26, 27]. Therefore, TTSPs have also been considered ideal candidate tumour markers. Transmembrane protease serine 3 (TMPRSS3) belongs to the TTSP family [28] and its encoded protein contains a transmembrane domain, a scavenger receptor cysteine-rich domain, an low-density lipoprotein receptorlike domain, and a serine protease transmembrane domain [29]. Currently, several studies have found that TMPRSS 3 is overexpressed in pancreatic cancer, gastric cancer, breast cancer, and nasopharyngeal carcinoma and plays an important biological role in the proliferation and invasion of related tumours [30-33]. However, both the expression level and clinical significance of TMPRSS3 in CRC remain unknown. In the present study, we found that TMPRSS3 was highly expressed in CRC radioresistant tissues through a comprehensive analysis of radio-processed CRC data sets, which are derived from public databases, and further investigated the underlying mechanism in the development of radioresistance. In addition, we also found that TMPRSS3 was upregulated in CRC tissues and identified its possible biological role in the development of CRC. This study fills a gap in extant research by studying the role of TMPRSS3 in the field of CRC research and provides new insights and directions for the development of more effective CRC therapeutic targets.

\section{Results}




\section{Results of Data Collection for the CRC Data Set}

Figure 1 and 2 depict the process of filtering the data set for our study. We obtained eight data sets related to radiation processing to explore the potential biological role of TMPRSS3 in the radiation resistance of $\mathrm{CRC}$, which comprised 198 radioresistant samples and 105 radiosensitive samples (Table 1). Moreover, 26 high-throughput data sets of $C R C$ tissues and paracancerous tissues met the inclusion criteria, including 1,130 CRC samples and 705 colorectal tissue samples derived from GEO. In addition, the normalized gene expression data of $471 \mathrm{CRC}$ tissue samples and 349 normal colorectal tissue samples were obtained from TCGA and GTEx databases to further expand the sample size (Table 2).

Table 1: Characteristics of the Radiation-Processed Datasets from the GEO Database in this Study.

\begin{tabular}{|cllll|}
\hline Publication year & Data source & Platform & Cancer group & Normal controls \\
\hline 2013 & GSE35452 & GPL570 & 22 & 24 \\
2020 & GSE119409 & GPL570 & 41 & 15 \\
2012 & GSE43206 & GPL6244 & 3 & 3 \\
2013 & GSE46862 & GPL6244 & 52 & 17 \\
2017 & GSE97543 & GPL13497 & 6 & 6 \\
2020 & GSE150082 & GPL13497 & 23 & 16 \\
2011 & GSE20298 & GPL4133 & 35 & 9 \\
2016 & GSE60331 & GPL15207 & 16 & 15 \\
\hline
\end{tabular}

Table 2: Characterization of Colorectal Cancer Microarrays from GEO Database in this Study. 


\begin{tabular}{|c|c|c|c|c|}
\hline Publication year & Data source & Platform & Cancer group & Normal controls \\
\hline 2011 & GSE24514 & GPL96 & 34 & 15 \\
\hline 2013 & GSE49355 & GPL96 & 20 & 18 \\
\hline 2015 & GSE68468 & GPL96 & 184 & 52 \\
\hline 2016 & GSE77953 & GPL96 & 17 & 6 \\
\hline 2018 & GSE110223 & GPL96 & 13 & 13 \\
\hline 2011 & GSE21815 & GPL6480 & 132 & 9 \\
\hline 2013 & GSE35279 & GPL6480 & 74 & 5 \\
\hline 2015 & GSE54986 & GPL10558 & 6 & 6 \\
\hline 2015 & GSE75548 & GPL10558 & 6 & 6 \\
\hline 2018 & GSE106582 & GPL10558 & 77 & 117 \\
\hline 2017 & GSE81558 & GPL15207 & 23 & 9 \\
\hline 2018 & GSE113513 & GPL15207 & 14 & 14 \\
\hline 2009 & GSE15781 & GPL2986 & 22 & 20 \\
\hline 2011 & GSE20842 & GPL4133 & 65 & 65 \\
\hline 2010 & GSE24713 & GPL11060 & 19 & 25 \\
\hline 2012 & GSE28000 & GPL1708 & 5 & 6 \\
\hline 2014 & GSE44076 & GPL13667 & 98 & 98 \\
\hline 2015 & GSE47063 & GPL6102 & 14 & 4 \\
\hline 2014 & GSE62321 & GPL97 & 20 & 18 \\
\hline 2017 & GSE87211 & GPL13497 & 203 & 160 \\
\hline 2017 & GSE103512 & GPL13158 & 57 & 12 \\
\hline 2018 & GSE113513 & GPL15207 & 14 & 14 \\
\hline 2020 & GSE141174 & GPL6104 & 3 & 3 \\
\hline 2020 & GSE151021 & GSE151021 & 4 & 4 \\
\hline 2020 & GSE156355 & GPL21185 & 6 & 6 \\
\hline NA & TCGA+GTEx & NA & 471 & 349 \\
\hline
\end{tabular}

Expression of TMPRSS3 is Upregulated in Radioresistant Tissues 
Through box plots, we intuitively observed that the expression level of TMPRSS3 in most radioresistant tissues was significantly higher than that in radiosensitive tissues (Fig. 3). ROCs further confirmed that the expression of TMPRSS3 in CRC radioresistant and radiosensitive tissues were significantly different (Fig. 4). Figure 5 depicts SROC $=0.71$ (95\% Cl: $0.67 \sim 0.75)$, with a pooled sensitivity of 0.42 (95\% Cl: 0.21 $\sim 0.66)$ and a pooled specificity of 0.91 (95\% Cl: $0.63 \sim 0.98)$. The above results suggest that TMPRSS3 has a good ability to distinguish radioresistant from radiosensitive tissues. Due to the small sample size of individual data sets, we integrated the TMPRSS3 expression data of all CRC radiation-related data sets in order to obtain reliable results. The fixed effects model was selected because of the small heterogeneity (I-squared $=0.0 \%$, p-value $=0.758)$. The SMD of TMPRSS3 was $0.38(95 \% \mathrm{Cl}: 0.14 \sim 0.63 ; \mathrm{p}$ value $<0.05$ ). The results of the sensitivity analysis revealed that the combined SMD was stable, and Begg's Test suggested that there was no publication bias among the included data sets (Fig. 6). This is the first study to demonstrate that the expression of TMPRSS3 is significantly higher in radioresistant tissues than in radiosensitive tissues of CRC. Thus, TMPRSS3 may play an important role in the biological process of $\mathrm{CRC}$ radioresistance.

\section{TMPRSS3 is Highly Expressed in CRC Tissues}

As is evident from Fig. 7, TMPRSS3 is highly expressed in most CRC tissues. Figure 8 suggests that TMPRSS3 has a good ability to distinguish CRC tissues from paracancerous tissues by ROC curves. Figure 9 presents SROC $=0.95$ (95\% Cl: $0.92 \sim 0.96)$, with a pooled sensitivity of 0.83 (95\% Cl: $0.70 \sim$ $0.91)$ and a pooled specificity of $0.92(95 \% \mathrm{Cl}: 0.88 \sim 0.95)$, and suggests that the discriminatory ability of TMPRSS3 has good specificity and sensitivity. To obtain comprehensive and reliable gene expression data for accurate and credible findings, we integrated the expression of TMPRSS3 across all data sets and conducted a meta-analysis. Due to the large heterogeneity among data sets (l-squared $=89.4 \%, \mathrm{p}$ value $=0.000)$, the random effects model was selected. The SMD was 1.55 (95\% Cl: $1.20 \sim 1.90 ; p$-value < 0.05). The sensitivity analysis revealed that the pooled result was stable, and Begg's Test verified that there was no publication bias among the included data sets, as depicted in Fig. 10. The above results confirmed that TMPRSS3 expression was significantly higher in CRC than in adjacent tissue.

\section{Relationship Between TMPRSS3 and CRC Metastasis}

The metastasis-prone property of CRC leads to its poorer prognosis of CRC than that of tumours in general. Therefore, in order to further explore the relevant mechanisms of CRC metastasis, we performed subgroup analysis of lymph node metastasis and distant metastasis.

In this study, eight data sets (GSE141174, GSE62321, GSE75548, GSE21815, GSE35279, GSE49355, GSE77953 and TCGA) were retrieved after eliminating the data sets that did not contain clinical information regarding lymph node metastasis. The meta-analysis using a fixed effect model (I-squared $=$ $0.0 \%, P=0.720)$ yielded SMD $=0.03(95 \% \mathrm{Cl}:-0.12 \sim 0.19 ; \mathrm{P}=0.669 ;$ Fig. $11 \mathrm{~A})$. There was no significant publication bias among the included studies (Begg's Test $\operatorname{Pr}>|z|=1.000$; Fig. 11B), and the sensitivity analysis indicated that the results were stable (Fig. 11C). Ultimately, we found that there was no statistical difference in TMPRSS3 expression among the subgroups of CRC lymph node metastases. 
We also investigated the relationship between TMPRSS3 and distant metastasis of CRC. A total of seven data sets (GSE87211, GSE62321, GSE81558, GSE21815, GSE35279, GSE49355, and TCGA) that included 154 distant metastatic CRC samples and 686 distant metastasis-free CRC samples were included according to the presence or absence of distant metastasis grouping. Meta-analysis (fixed effect model; Isquared $=10.9 \%, p=0.346)$ indicated $S M D=-0.10$ (95\% Cl: $-0.28 \sim 0.09 ; p=0.310 ;$ Fig. 11D). Begg's Test suggested no publication bias ( $\operatorname{Pr}>|z|=0.707$; Fig. 11E), and sensitivity analysis revealed stable SMD results. The results suggested that TMPRSS3 had no statistical difference among subgroups with distant metastasis in CRC (Fig. 11F).

\section{High TMPRSS3 Expression Indicates Poor Prognosis in CRC Patients}

To investigate the link between TMPRSS3 expression and the prognosis of CRC patients, 1221 samples containing overall survival (OS) data and 790 samples containing disease-free survival (DFS) data from GEO and TCGA databases were included. The median TMPRSS3 expression value of each data set was used as the cut-off value to classify the data sets into high and low expression groups. The Kaplan-Meier method was used for prognostic analysis of OS in Fig. 12 and DFS in Fig. 13. Simultaneously, we performed COX analysis of survival data and calculated the HR. The COX results for DFS and OS are presented in Tables 3 and 4, respectively. Further, a pooled analysis of HR values revealed that TMPRSS3 was associated with poor DFS prognosis in CRC patients (Fig. 14A). However, the pooled HR value for OS suggested no statistical significance, which we believe may be due to the small sample size (Fig. 14B). In future studies, a larger sample will be required to further verify its accuracy.

Table 3: Characteristics of the DFS Datasets from the GEO Database in this Study.

\begin{tabular}{lrllc}
\hline Publication year & Data source & HR & 95\%CI_lower & 95\%CI_upper \\
\hline 2010 & GSE14333 & 1.395 & 1.034 & 1.881 \\
2009 & GSE17537 & 0.7285 & 0.2923 & 1.816 \\
2011 & GSE24549 & 1.628 & 0.8831 & 3.002 \\
2011 & GSE24550 & 0.963 & 0.3909 & 2.372 \\
2014 & GSE38832 & 0.9298 & 0.2492 & 3.469 \\
2015 & GSE56699 & 4.974 & 1.074 & 23.03 \\
2017 & GSE87211 & 0.9468 & 0.5405 & 1.659 \\
\hline
\end{tabular}

Table 4: Characteristics of the OS Datasets from the GEO Database in this Study.

\begin{tabular}{lllll}
\hline Publication year & Data source & HR & 95\%CI_lower & 95\%CI_upper \\
\hline NA & TCGA & 1.112 & 0.7816 & 1.582 \\
2009 & GSE12945 & 1.448 & 0.459 & 4.566 \\
2009 & GSE17537 & 0.6424 & 0.2624 & 1.573 \\
2017 & GSE71187 & 0.9516 & 0.4122 & 2.197 \\
2017 & GSE72970 & 1.268 & 0.7901 & 2.035 \\
2017 & GSE87211 & 1.323 & 0.6071 & 2.884 \\
2017 & GSE103479 & 1.068 & 0.6424 & 1.775 \\
\hline
\end{tabular}




\section{GO and KEGG Enrichment Analysis Results of TMPRSS3 and Positively Correlated Genes}

After Pearson's correlation analysis of 18 CRC data sets derived from GEO, TCGA, and GTEx public databases, 429 positively expressed genes-including TRIM29, SKAP1, BATF, PRSS22, RASAL1, SPTBN5, and C10orf81-were obtained for the next step of bioinformatics analysis to explore the potential function and mechanism of TMPRSS3 during CRC progression. These positively expressed genes can be considered to act synergistically with TMPRSS3.

To understand the biological functions of TMPRSS3 and its positively expressed genes, we performed GO and KEGG pathway enrichment analysis on 429 positively correlated genes. The results of $\mathrm{GO}$ analysis showed that in BP, the abovementioned genes were mainly enriched in the cell-cell adhesion, extracellular matrix organization, cell cycle, extracellular matrix disassembly, peptidyl-tyrosine dephosphorylation, single cell-organism cell adhesion, and negative regulation of endopeptidase signalling activity (Fig. 15A). In CC, the abovementioned genes were mainly enriched in plasma membrane, cytosol, extracellular exosome, extracellular space, cell surface, cell-cell adherens junction, cell-cell junction, and membrane raft (Fig. 15B). In MF, TMPRSS3 and its positively correlated genes were mainly enriched in protein binding, serine-type endopeptidase activity, protein tyrosine phosphatase activity, SUMO activity, and serine-type endopeptidase inhibitor activity (Fig. 15C). Furthermore, KEGG signalling pathway enrichment analysis revealed that TMPRSS3 and its positively correlated genes were enriched in proteasome and ECM-receptor interaction pathways (Fig. 15D). Subsequently, the PPI network was constructed for the above positively expressed genes using STRING and screened using CentiScape, thereby resulting in a total of four genes with high connectivity: MSLN, MFI2, CKAP4, and PXN (Fig. 15E). These genes may interact with TMPRSS3 and participate in the regulation of proliferation, migration, and apoptosis of CRC cells.

\section{Discussion}

Through this study, we are the first to find that TMPRSS3 is highly expressed in radioresistant CRC tissues based on TCGA, GEO, and GTEx data sets as well as published studies. Simultaneously, we also confirmed the abnormal expression of TMPRSS 3 in CRC tissues by comparing the mRNA expression profiles of cancer tissues and paracancerous tissues. Furthermore, we analysed the effect of TMPRSS3 on the prognosis of CRC patients. CRC survival data from public databases were analysed using the Kaplan-Meier method and COX. The HR was subsequently pooled and the results revealed that the upregulated expression of TMPRSS3 was a risk factor for poor prognosis in CRC. In addition, we explored the key biological processes and pathways involved in TMPRSS3 during CRC tumorigenesis, invasion, metastasis, and resistance to radiotherapy by GO analysis and KEGG pathway annotation. Finally, it was confirmed that TMPRSS3 has potential as a biomarker and therapeutic target for CRC.

As a member of TTSPs, TMPRSS3 plays an important role in the growth, metastasis, invasion, and digestion of the extracellular matrix of cancer cells and promotes tumour progression through proteolytic 
activity $[34,35]$. Currently, there are a few studies on the mechanism of TMPRSS3 in tumours and only a few of them have reported the mechanism of TMPRSS3 in the process of tumour tumorigenesis and progression. Wallrapp et al. (2020) found that TMPRSS3 expression was upregulated in the pancreatic cancer cell line SUIT-2 and was associated with the invasion and metastasis of cancer cells [32]. In gastric cancer, the knockdown of TMPRSS3 expression affected ERK1/2 and PI3K/Akt pathways and ultimately inhibited gastric cancer cell proliferation, invasion, and epithelial-mesenchymal transition (EMT) [30]. In addition, Rui et al. (2015) found that TMPRSS3 was significantly highly expressed in breast cancer, and a high expression of TMPRSS3 was associated with poor prognosis [31]. Furthermore, in nasopharyngeal carcinoma, the knockdown of TMPRSS3 led to the inactivation of the PI3K/Akt signalling pathway and then led to the inhibition of the proliferation, migration, and invasion of NPC cells [33]. The abovementioned studies indicate that TMPRSS3 has potential as a treatment for a variety of malignancies. However, no study on TMPRSS3 in CRC has been reported thus far.

This study was the first to find that TMPRSS3 expression was upregulated in CRC tissues and negatively correlated with DFS in CRC patients. GO analysis revealed that TMPRSS3 mainly functioned in cellular components-such as extracellular space, cell surface, cell-adherens junction, and cell-cell junction-and was involved in biological processes or molecular function, such as extracellular matrix disassembly and cadherin binding involved in cell-cell adhesion. Extracellular matrix (ECM), a major part of the extracellular microenvironment, is the place of cell survival and activity. Recent studies have revealed that ECM remodelling has an important impact on tumour invasion and metastasis [36]. E-cadherin is a calcium-dependent cell adhesin that mainly mediates homogenous cell-cell adhesion and is involved in forming and maintaining normal cell-cell junctions. The downregulation or loss of E-cadherin expression can cause EMT, thereby leading to epithelial tumour invasion and metastasis [37]. Therefore, we believe that the expression product of TMPRSS3 in CRC, as a protein-degrading enzyme, can digest the extracellular matrix through the hydrolysis of proteases, create a relaxed microenvironment for the proliferation, lead to the differentiation and metastasis of tumour cells, and lead cancer cells to break through the basement membrane and invade the surrounding tissues. Simultaneously, TMPRSS3 breaks down E-cadherin, which reduces adhesion among tumour cells and promotes detachment and metastasis of tumour cells from the primary tumour. Moreover, TMPRSS3 may serve as a potential biomarker to identify CRC tumours with more aggressive characteristics and may also be a prognostic tool and a potential therapeutic target for future CRC patients.

Radiotherapy, as a local treatment, induces cancer cell senescence, apoptosis, necrosis, autophagy, and mitotic catastrophe by ionizing radiation generated by high-energy rays, rivalling surgery in the efficacy of certain tumours [7]. However, certain patients remain insensitive to radiotherapy. Therefore, exploring the mechanism of radiotherapy resistance in CRC is significant in improving the efficacy of radiotherapy. Through this study, we are the first to find that the expression of TMPRSS3 in CRC radioresistant tissues is significantly higher than that in radiosensitive tissues. Previous studies have revealed that TMPRSS 3 is positively associated with the PI3K/Akt signalling pathway in gastric cancer and nasopharyngeal carcinoma, and TMPRSS 3 can promote tumour progression by activating the PI3K/Akt/ERK1/2 signalling pathway [30,33]. Furthermore, the GO analysis in this study also suggested that TMPRSS3 
could affect serine-type endopeptidase activity and threonine-type endopeptidase activity. Takeuchi et al. (2005) found that the autophagy pathway is negatively regulated by the PI3K/Akt/mTOR pathway [38]. Autophagy is a programmed death process in which senescent organelles or macromolecules are degraded into amino acids and energy for recycling in lysosomes [39]. It has been shown that increasing autophagy in tumour cells, to a certain extent, can enhance the effect of radiation therapy [40]. Therefore, we suggest that the upregulation of TMPRSS3 expression in CRC activates the PI3K/Akt/mTOR pathway and inhibits autophagy, which in turn plays a role in radioresistance.

In addition, we also screened four hub genes-MSLN, MFI2, CKAP4, and PXN. These four genes may play an important role in concert with TMPRSS3 in the process of CRC occurrence, invasion, and metastasis. Cytoskeleton associated protein 4 (CKAP4) is a type II transmembrane protein [41]. Currently, CKAP4 has been found to be involved in a variety of signalling pathways and is closely related to the occurrence and migration of several tumours [42]. Kimura et al. (2016) found that DKK 1/CKAP4 signalling activates AKT by forming a complex between the proline-rich domain of CKAP4 and the Src homology 3 domain of $\mathrm{PI} 3 \mathrm{~K}$, thereby resulting in the proliferation of both normal and cancer cells. Anti-CKAP4 antibodies have been used to block DKK 1 binding to CKAP4, inhibit AKT activity in cancer cell lines, and attenuate tumour formation [43]. Therefore, we conclude that a high expression of CKAP4 leading to ATK activation also inhibits autophagy in CRC cells and synergizes with TMPRSS3 to exert radioresistance. Distant metastasis is an important factor in the poor prognosis of CRC patients. Mesothelin (MSLN), a glycoprotein attached to cell membranes by glycosylphosphatidylinositol, is highly expressed in lung, ovarian, and pancreatic cancers [44, 45]. Furthermore, Tozbikian et al. (2014) found that patients with positive MSLN in triple negative breast carcinomas (TNBC) were more likely to develop metastases than those with negative MSLN [46]. The possible involvement of MSLN in cell adhesion in combination with CA125 was first revealed by Chang et al. (1996)[47]. Paxillin (PXN) encodes a phosphoprotein on the cytoskeleton and acts as an adaptor protein of the integrin signalling pathway, which regulates signal transduction from the extracellular matrix into cells through the integrin signalling pathway and plays a key role in the adhesion, metastasis, and invasion of tumour cells [48]. Lee et al. (2010) found that a knockdown of PXN expression resulted in decreased radiation-induced cell adhesion and promoted cell death in SW480 colon cancer cells [49]. In summary, based on the important role of MSLN and PXN in the adhesion process, we believe that TMPRSS3 can cooperate with MSLN and PXN to regulate cancer cell adhesion and, thus, promote the metastasis and spread of CRC cancer cells. Melanotransferrin (MFI2) encodes a glycoprotein of the transferrin family that is involved in a variety of cellular biological processes, such as iron metabolism, proenzyme activation, eosinophil differentiation, tumorigenesis, and tumour cell proliferation and migration [50,51]. Shin et al. (2014) found that MFI2 was highly expressed in CRC and had a high specificity for diagnosis [51]. Thus, MFI2 is expected to be a new marker for diagnosing CRC.

In conclusion, for the first time, TMPRSS3 expression was found to be upregulated in CRC radioresistant tissues and CRC tissues and was negatively correlated with CRC prognosis. Furthermore, TMPRSS3 synergizes with MSLN, MFI2, CKAP4, PXN, and other genes to play an important role in CRC tumorigenesis, invasion, metastasis, and radioresistance; however, the specific pathway remains to be 
verified by additional cell and animal experiments. Although our study has a few limitations, such as the lack of cell experiments and animal experiments, the results still strongly suggest that TMPRSS3 has potential as a new biomarker and therapeutic target for CRC and has a few implications for predicting the prognosis of CRC patients. Further studies on TMPRSS3 will likely enhance the existing knowledge of CRC tumorigenesis, invasion, metastasis, and radioresistance.

\section{Conclusion}

TMPRSS3 is highly expressed in CRC radioresistant tissues and CRC tissues; it is also found to be negatively correlated with the prognosis of CRC patients. Thus, TMPRSS3 has potential as a biomarker and therapeutic target for CRC.

\section{Materials And Methods}

\section{Collecting TMPRSS3 mRNA Expression Data and Radiotherapy-related Data in Colorectal Cancer}

The TMPRSS3 mRNA gene expression data and clinicopathological information of this study were obtained from GEO, ArrayExpress, TCGA, and GTEx public databases. The following search strategy of the database was applied: (colon OR rectal OR colorectal) AND cancer. In addition, chips or sequencing data related to CRC radiation processing were also obtained from the above databases to investigate the function of TMPRSS3 in CRC radioresistance. Furthermore, the search strategy was to add (radio OR radiotherapy OR radiation). The search cut-off date for the abovementioned data was 31 May 2021.

\section{Processing High-throughput Data in Colorectal Cancer}

All data sets included in this study were downloaded and collated by the GEO query (2.54) package of R 4.0.5 software. The chips or sequencing data were collected in the following processing criteria: (1) all unnormalized genes were log2 transformed; (2) when multiple probes in the same chip corresponded to the same gene, the median expression value was selected; (3) the missing values in the data sets were replaced by the results calculated by the k-nearest neighbours $(K N N)$; (4) the data of the same gene chip platform were combined and the batch effect was eliminated by the combat algorithm of the sva (3.38.0) package of R 4.0.5 software.

\section{Analysing the Expression of TMPRSS3 in CRC Radioresistant Tissues}

In order to visually demonstrate the expression of TMPRSS3 in CRC radioresistant tissues, the ggplot2 (3.3.3) package of R 4.0.5 software was used to visualize the expression of TMPRSS3 in individual data sets. 
Due to the small sample size of individual data sets and in order to obtain more reliable results, we conducted a pooled analysis of TMPRSS3 expression in CRC radioresistant tissues in this study. Metaanalysis was performed using Stata16.0 software to calculate the standardized mean difference (SMD) and forest plots were drawn. In addition, publication bias was examined using a linear regression model (Begg's test), and the magnitude of heterogeneity in the pooled analysis was measured using l-squared and $p$-value. A fixed effect model was used if I-squared $\leq 30 \%$ and $p$-value $>0.05$, thereby indicating good homogeneity between the results. Conversely, a random-effects model was used if I-squared $\geq 30 \%$ and p-value $<0.05$.

Furthermore, to investigate the ability of TMPRSS3 to distinguish radioresistant and radiosensitive CRC tissues, we performed the receiver operating characteristic curves (ROC) analysis using the R package pROC (1.17.0.1), and the area under the curve (AUC) was used to measure the discriminatory ability of TMPRSS3 for different groups. The summary receiver operating characteristic curve (SROC), specificity, and sensitivity were also calculated using the STATA16.0 software.

\section{Analysing TMPRSS3 Expression in CRC Tissues}

In the same manner, we also used STATA 16.0 and R 4.0.5 software to comprehensively analyse the expression level of TMPRSS3 in CRC tissues, plotted box plots, ROC curves, and SROC curves and further calculated parameters such as AUC, specificity, and sensitivity [52].

\section{Exploring the Correlation between TMPRSS3 Expression and Clinical Pathology}

Metastasis is the leading cause of death in CRC patients. To further explore the relationship between TMPRSS3 and CRC metastasis, we screened data sets containing clinical information pertaining to lymph node metastasis and distant metastasis, then grouped TMPRSS3 expression values according to lymph node metastasis and distant metastasis, and calculated SMD values using Stata 16.0.

\section{Evaluating the Effect of TMPRSS3 on the Prognosis of CRC Patients}

In order to investigate whether TMPRSS3 can be used as a prognostic indicator, we obtained CRC data sets that included survival time through GEO and TCGA databases; the survival time was converted into months for standardization. The median of TMPRSS3 expression values in each data set was used as a cut-off value, and the included data were divided into high and low expression groups according to this cut-off value. Then, the Kaplan-Meier method was used to analyse the relationship between TMPRSS3 and CRC prognosis, and the log-rank test was used for comparison. A p-value $<0.05$ was considered statistically significant. Subsequently, we further analysed the effect of TMPRSS3 on the prognosis of CRC patients through a COX analysis and pooled the hazard ratio (HR) using Stata 16.0 software.

\section{Obtaining Co-expressed Genes of TMPRSS3 in CRC}


To further explore the potential biological function of TMPRSS3 during CRC progression, we conducted Pearson's correlation analysis on all the included data sets through the cor.test 0 function of R 4.0.5 software to obtain positive correlation genes. Pearson's correlation coefficient, also known as Pearson's product-moment correlation coefficient, is a linear correlation coefficient and is used to reflect the degree of linear correlation between two variables. It is expressed by the correlation coefficient ' $r$ '. The larger the absolute value of $r$, the stronger the correlation. The following screening criteria were employed in this study: $r>0$; $p$-value $<0.05$. Genes that appeared five times or more in all data sets were selected as positively expressed genes of TMPRSS3 for further bioinformatics analysis.

\section{Exploring the Potential Biological Functions and Signalling Pathways of TMPRSS3 in CRC}

ClusterProfiler is a package developed based on the R language, which enables GO and KEGG enrichment analysis and visualization of genes and gene clusters. In this study, clusterProfiler (3.16.1) was used to perform functional and signalling pathway enrichment analysis of TMPRSS3 and its positively correlated genes, including (1) GO functional enrichment analysis (cellular component (CC), molecular function (MF), and biological process (BP)) and (2) KEGG signalling pathway enrichment analysis.

STRING is a database of known and predicted protein-protein interactions. We used it to construct the protein-protein interaction network (PPI network) of co-expressed genes (high confidence score $=0.700$ ). The PPI network was screened by the Cytoscape 3.8.0 software. Cytoscape is an open-source network visualization and analysis software based on Java. Cytoscape can be used to perform large-scale analysis of protein-protein interactions, protein-DNA, and genetic interactions in a visualized environment. Subsequently, in order to further explore the interaction between TMPRSS3 and its co-expressed genes, we calculated the degree centrality of each node in the PPI network by utilizing the CentiScape 2.2 plugin of Cytoscape. Furthermore, we determined the hub genes in accordance with the numerical magnitude of the degree centrality.

\section{Declarations}

\section{Acknowledgments}

The authors gratefully acknowledge the Gene Expression Omnibus (GEO) database, the Cancer Genome Atlas (TCGA) database, ArrayExpress database, and GTEx database, which made the data available.

\section{Authors' contributions}

$\mathrm{GC}, \mathrm{RH}, \mathrm{RW}$ and $\mathrm{XQ}$ jointly proposed the study concept and designed the study protocol. JW and $\mathrm{HH}$ contributed to acquisition of data and data analysis. YF, DZ and WH edited and revised the manuscript. All authors read and approved the final manuscript. 


\section{Funding}

This work was supported by Guangxi Zhuang Autonomous Region Health Commission Self-Financed Scientific Research Project (Z20210442).

\section{Availability of data and materials}

The raw data for this study are obtained from the TCGA database, GEO database, ArrayExpress database, and GTEx database. All databases are publicly available.

\section{Ethics approval and consent to participate}

Not necessary.

\section{Consent for publication}

Not applicable.

\section{Competing interests}

No potential conflict of interest was reported by the authors.

\section{References}

1. Huo JF, Chen XB: Knockdown of TMPRSS3 inhibits cell proliferation, migration/invasion and induces apoptosis of glioma cells. J Cell Biochem 2018.

2. Luo P, Lu G, Fan LL, Zhong X, Yang H, Xie R, Lv Z, Lv QZ, Fu D, Yang LX, Ma Y: Dysregulation of TMPRSS3 and TNFRSF11B correlates with tumorigenesis and poor prognosis in patients with breast cancer. Oncol Rep 2017, 37(4):2057-2062.

3. Pelkonen M, Luostari K, Tengström M, Ahonen H, Berdel B, Kataja V, Soini Y, Kosma VM, Mannermaa A: Low expression levels of hepsin and TMPRSS 3 are associated with poor breast cancer survival. BMC Cancer 2015, 15:431.

4. Sung H, Ferlay J, Siegel RL, Laversanne M, Soerjomataram I, Jemal A, Bray F: Global Cancer Statistics 2020: GLOBOCAN Estimates of Incidence and Mortality Worldwide for 36 Cancers in 185 Countries. $C A$ Cancer J Clin 2021, 71(3):209-249. 
5. Dekker E, Tanis PJ, Vleugels JLA, Kasi PM, Wallace MB: Colorectal cancer. Lancet 2019, 394(10207):1467-1480.

6. Provenzale D, Ness RM, Llor X, Weiss JM, Abbadessa B, Cooper G, Early DS, Friedman M, Giardiello FM, Glaser K, et al: NCCN Guidelines Insights: Colorectal Cancer Screening, Version 2.2020. J Nat/ Compr Canc Netw 2020, 18(10):1312-1320.

7. Kim BM, Hong Y, Lee S, Liu P, Lim JH, Lee YH, Lee TH, Chang KT, Hong Y: Therapeutic Implications for Overcoming Radiation Resistance in Cancer Therapy. Int J Mol Sci 2015, 16(11):26880-26913.

8. [Chinese Protocol of Diagnosis and Treatment of Colorectal Cancer (2020 edition)]. Zhonghua Wai Ke Za Zhi 2020, 58(8):561-585.

9. Hashiguchi Y, Muro K, Saito Y, Ito Y, Ajioka Y, Hamaguchi T, Hasegawa K, Hotta K, Ishida H, Ishiguro M, et al: Japanese Society for Cancer of the Colon and Rectum (JSCCR) guidelines 2019 for the treatment of colorectal cancer. Int J Clin Oncol 2020, 25(1):1-42.

10. Miller KD, Nogueira L, Mariotto AB, Rowland JH, Yabroff KR, Alfano CM, Jemal A, Kramer JL, Siegel RL: Cancer treatment and survivorship statistics, 2019. CA Cancer J Clin 2019, 69(5):363-385.

11. Abraha I, Aristei C, Palumbo I, Lupattelli M, Trastulli S, Cirocchi R, De Florio R, Valentini V: Preoperative radiotherapy and curative surgery for the management of localised rectal carcinoma. Cochrane Database Syst Rev 2018, 10(10):Cd002102.

12. Petrelli F, Trevisan F, Cabiddu M, Sgroi G, Bruschieri L, Rausa E, Ghidini M, Turati L: Total Neoadjuvant Therapy in Rectal Cancer: A Systematic Review and Meta-analysis of Treatment Outcomes. Ann Surg 2020, 271(3):440-448.

13. Smith CA, Kachnic LA: Evolving Role of Radiotherapy in the Management of Rectal Carcinoma. Surg Oncol Clin N Am 2017, 26(3):455-466.

14. Baptistella AR, Landemberger MC, Dias MVS, Giudice FS, Rodrigues BR, da Silva P, Cassinela EK, Lacerda TC, Marchi FA, Leme AFP, et al: Rab5C enhances resistance to ionizing radiation in rectal cancer. J Mol Med (Berl) 2019, 97(6):855-869.

15. Emons G, Spitzner M, Reineke S, Möller J, Auslander N, Kramer F, Hu Y, Beissbarth T, Wolff HA, RaveFränk $\mathrm{M}$, et al: Chemoradiotherapy Resistance in Colorectal Cancer Cells is Mediated by Wnt/ $\beta$-catenin Signaling. Mol Cancer Res 2017, 15(11):1481-1490.

16. Ferrandon S, DeVecchio J, Duraes L, Chouhan H, Karagkounis G, Davenport J, Orloff M, Liska D, Kalady MF: CoA Synthase (COASY) Mediates Radiation Resistance via PI3K Signaling in Rectal Cancer. Cancer Res 2020, 80(2):334-346. 
17. Yang J, Lin Y, Huang Y, Jin J, Zou S, Zhang X, Li H, Feng T, Chen J, Zuo Z, et al: Genome landscapes of rectal cancer before and after preoperative chemoradiotherapy. Theranostics 2019, 9(23):6856-6866.

18. Misiakos EP, Karidis NP, Kouraklis G: Current treatment for colorectal liver metastases. World J Gastroenterol 2011, 17(36):4067-4075.

19. Di W, Weinan X, Xin L, Zhiwei Y, Xinyue G, Jinxue T, Mingqi L: Long noncoding RNA SNHG14 facilitates colorectal cancer metastasis through targeting EZH2-regulated EPHA7. Cell Death Dis 2019, 10(7):514.

20. Olsen LM, Fiehn AK, Hasselby JP: ERCC1 expression in advanced colorectal cancer and matched liver metastases. Pathol Res Pract 2020, 216(3):152826.

21. Pasetto LM, Jirillo A, ladicicco G, Rossi E, Paris MK, Monfardini S: FOLFOX versus FOLFIRI: a comparison of regimens in the treatment of colorectal cancer metastases. Anticancer Res 2005, 25(1b):563-576.

22. Van Cutsem E, Cervantes A, Nordlinger B, Arnold D: Metastatic colorectal cancer: ESMO Clinical Practice Guidelines for diagnosis, treatment and follow-up. Ann Oncol 2014, 25 Suppl 3:iii1-9.

23. Davies JM, Goldberg RM: Treatment of metastatic colorectal cancer. Semin Oncol 2011, 38(4):552560.

24. Zhu D, Ren L, Xu J: [Interpretation of guidelines for the diagnosis and comprehensive treatment of colorectal cancer liver metastases in China(v2013)]. Zhonghua Wei Chang Wai Ke Za Zhi 2014, 17(6):525-529.

25. Murray AS, Hyland TE, Sala-Hamrick KE, Mackinder JR, Martin CE, Tanabe LM, Varela FA, List K: The cell-surface anchored serine protease TMPRSS13 promotes breast cancer progression and resistance to chemotherapy. Oncogene 2020, 39(41):6421-6436.

26. Tanabe LM, List K: The role of type II transmembrane serine protease-mediated signaling in cancer. FEBS J 2017, 284(10):1421-1436.

27. Varela FA, Foust VL, Hyland TE, Sala-Hamrick KE, Mackinder JR, Martin CE, Murray AS, Todi SV, List K: TMPRSS13 promotes cell survival, invasion, and resistance to drug-induced apoptosis in colorectal cancer. Sci Rep 2020, 10(1):13896.

28. Jiang W, Peng A, Chen Y, Pang B, Zhang Z: Long non-coding RNA EBLN3P promotes the recovery of the function of impaired spiral ganglion neurons by competitively binding to miR-204-5p and regulating TMPRSS3 expression. Int J Mol Med 2020, 45(6):1851-1863.

29. Guipponi M, Antonarakis SE, Scott HS: TMPRSS3, a type Il transmembrane serine protease mutated in non-syndromic autosomal recessive deafness. Front Biosci 2008, 13:1557-1567. 
30. Li SL, Chen X, Wu T, Zhang XW, Li H, Zhang Y, Ji ZZ: Knockdown of TMPRSS3 inhibits gastric cancer cell proliferation, invasion and EMT via regulation of the ERK1/2 and PI3K/Akt pathways. Biomed Pharmacother 2018, 107:841-848.

31. Rui X, Li Y, Jin F, Li F: TMPRSS3 is a novel poor prognostic factor for breast cancer. Int J Clin Exp Pathol 2015, 8(5):5435-5442.

32. Wallrapp C, Hähnel S, Müller-Pillasch F, Burghardt B, Iwamura T, Ruthenbürger M, Lerch MM, Adler G, Gress TM: A novel transmembrane serine protease (TMPRSS3) overexpressed in pancreatic cancer. Cancer Res 2000, 60(10):2602-2606.

33. Wang JY, Jin X, Li XF: Knockdown of TMPRSS3, a Transmembrane Serine Protease, Inhibits Proliferation, Migration, and Invasion in Human Nasopharyngeal Carcinoma Cells. Oncol Res 2018, 26(1):95-101.

34. Dorn J, Beaufort N, Schmitt M, Diamandis EP, Goettig P, Magdolen V: Function and clinical relevance of kallikrein-related peptidases and other serine proteases in gynecological cancers. Crit Rev Clin Lab Sci 2014, 51(2):63-84.

35. Hooper JD, Clements JA, Quigley JP, Antalis TM: Type II transmembrane serine proteases. Insights into an emerging class of cell surface proteolytic enzymes. J Biol Chem 2001, 276(2):857-860.

36. Egeblad M, Rasch MG, Weaver VM: Dynamic interplay between the collagen scaffold and tumor evolution. Curr Opin Cell Biol 2010, 22(5):697-706.

37. Shen T, Zhang K, Siegal GP, Wei S: Prognostic Value of E-Cadherin and $\beta$-Catenin in Triple-Negative Breast Cancer. Am J Clin Pathol 2016, 146(5):603-610.

38. Takeuchi H, Kondo Y, Fujiwara K, Kanzawa T, Aoki H, Mills GB, Kondo S: Synergistic augmentation of rapamycin-induced autophagy in malignant glioma cells by phosphatidylinositol 3-kinase/protein kinase B inhibitors. Cancer Res 2005, 65(8):3336-3346.

39. Li YJ, Lei YH, Yao N, Wang CR, Hu N, Ye WC, Zhang DM, Chen ZS: Autophagy and multidrug resistance in cancer. Chin J Cancer 2017, 36(1):52.

40. Galluzzi L, Bravo-San Pedro JM, Demaria S, Formenti SC, Kroemer G: Activating autophagy to potentiate immunogenic chemotherapy and radiation therapy. Nat Rev Clin Oncol 2017, 14(4):247-258.

41. Vedrenne $C$, Hauri HP: Morphogenesis of the endoplasmic reticulum: beyond active membrane expansion. Traffic 2006, 7(6):639-646.

42. Kikuchi A, Fumoto K, Kimura H: The Dickkopf1-cytoskeleton-associated protein 4 axis creates a novel signalling pathway and may represent a molecular target for cancer therapy. Br J Pharmacol 2017, 174(24):4651-4665. 
43. Kimura H, Fumoto K, Shojima K, Nojima S, Osugi Y, Tomihara H, Eguchi H, Shintani Y, Endo H, Inoue $\mathrm{M}$, et al: CKAP4 is a Dickkopf1 receptor and is involved in tumor progression. J Clin Invest 2016, 126(7):2689-2705.

44. Morello A, Sadelain M, Adusumilli PS: Mesothelin-Targeted CARs: Driving T Cells to Solid Tumors. Cancer Discov 2016, 6(2):133-146.

45. Zhenjiang L, Rao M, Luo X, Sandberg E, Bartek J, Jr., Schoutrop E, von Landenberg A, Meng Q, Valentini D, Poiret T, et al: Mesothelin-specific Immune Responses Predict Survival of Patients With Brain Metastasis. EBioMedicine 2017, 23:20-24.

46. Tozbikian G, Brogi E, Kadota K, Catalano J, Akram M, Patil S, Ho AY, Reis-Filho JS, Weigelt B, Norton L, et al: Mesothelin expression in triple negative breast carcinomas correlates significantly with basal-like phenotype, distant metastases and decreased survival. PLoS One 2014, 9(12):e114900.

47. Chang K, Pastan I: Molecular cloning of mesothelin, a differentiation antigen present on mesothelium, mesotheliomas, and ovarian cancers. Proc Natl Acad Sci U S A 1996, 93(1):136-140.

48. López-Colomé AM, Lee-Rivera I, Benavides-Hidalgo R, López E: Paxillin: a crossroad in pathological cell migration. J Hematol Oncol 2017, 10(1):50.

49. Lee M, Park JJ, Lee YS: Adhesion of ST6Gal I-mediated human colon cancer cells to fibronectin contributes to cell survival by integrin beta1-mediated paxillin and AKT activation. Oncol Rep 2010, 23(3):757-761.

50. Hernández-Pasos J, Valentín-Tirado G, García-Arrarás JE: Melanotransferrin: New Homolog Genes and Their Differential Expression during Intestinal Regeneration in the Sea Cucumber Holothuria glaberrima. J Exp Zool B Mol Dev Evol 2017, 328(3):259-274.

51. Shin J, Kim HJ, Kim G, Song M, Woo SJ, Lee ST, Kim H, Lee C: Discovery of melanotransferrin as a serological marker of colorectal cancer by secretome analysis and quantitative proteomics. $J$ Proteome Res 2014, 13(11):4919-4931.

52. Liang L, Zhao K, Zhu JH, Chen G, Qin XG, Chen JQ: Comprehensive evaluation of FKBP10 expression and its prognostic potential in gastric cancer. Oncol Rep 2019, 42(2):615-628.

\section{Figures}




\section{Identification of studies via databases and registers}

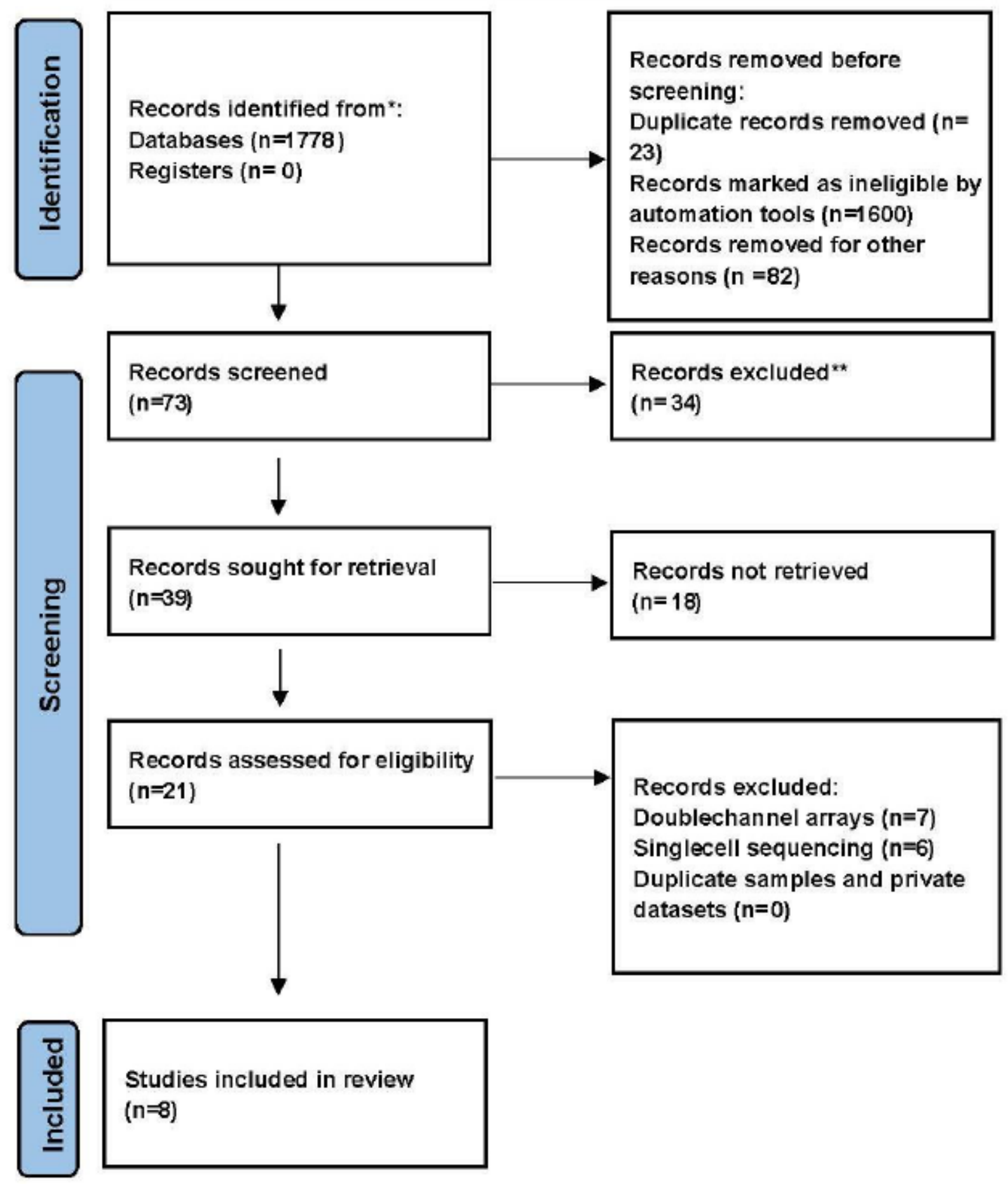

Figure 1

Screening process for radiation-processed CRP mRNA data sets from Gene Expression Omnibus (GEO). 


\section{Identification of studies via databases and registers}

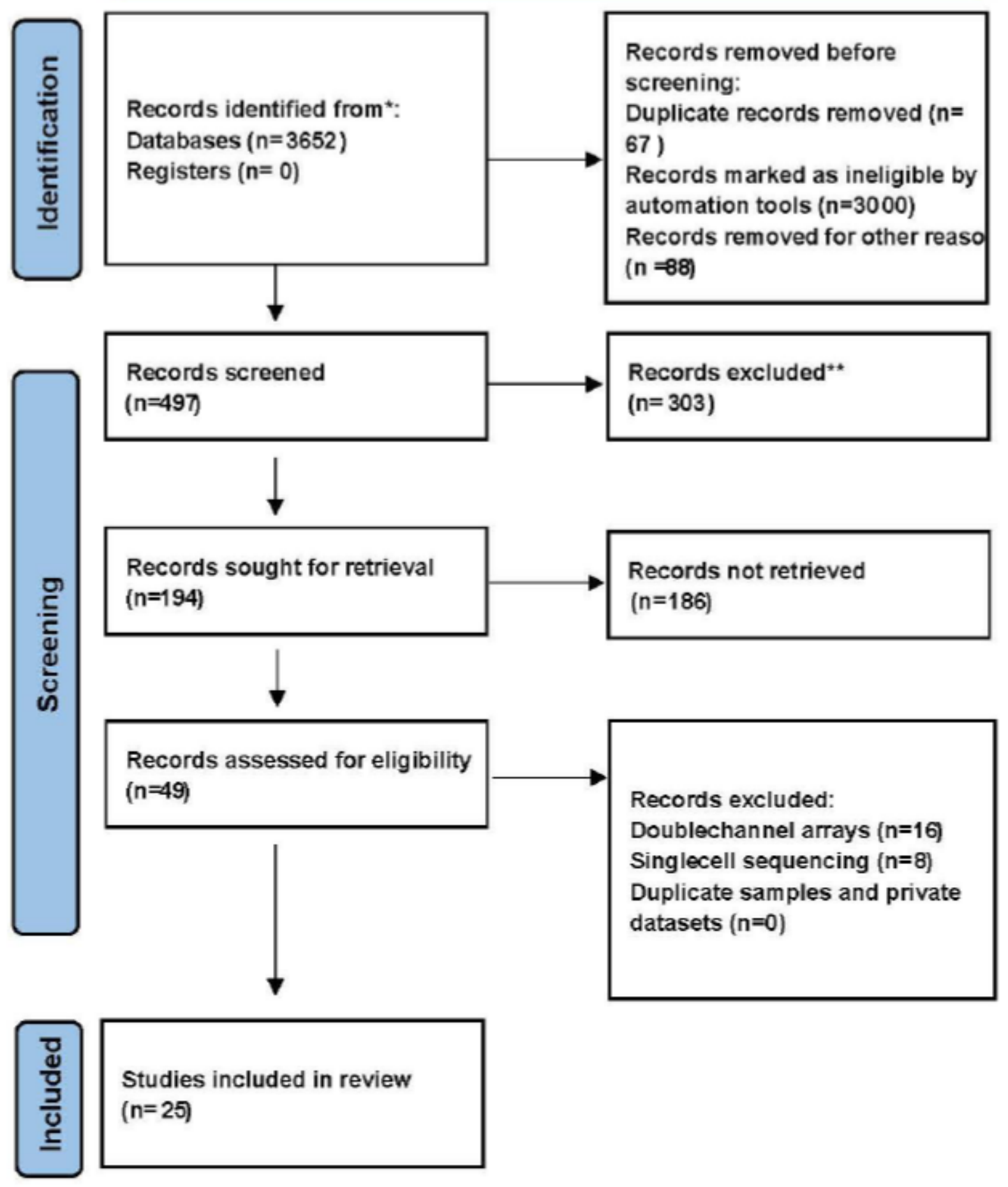

Figure 2

Screening process for CRC mRNA data sets from Gene Expression Omnibus (GEO). 


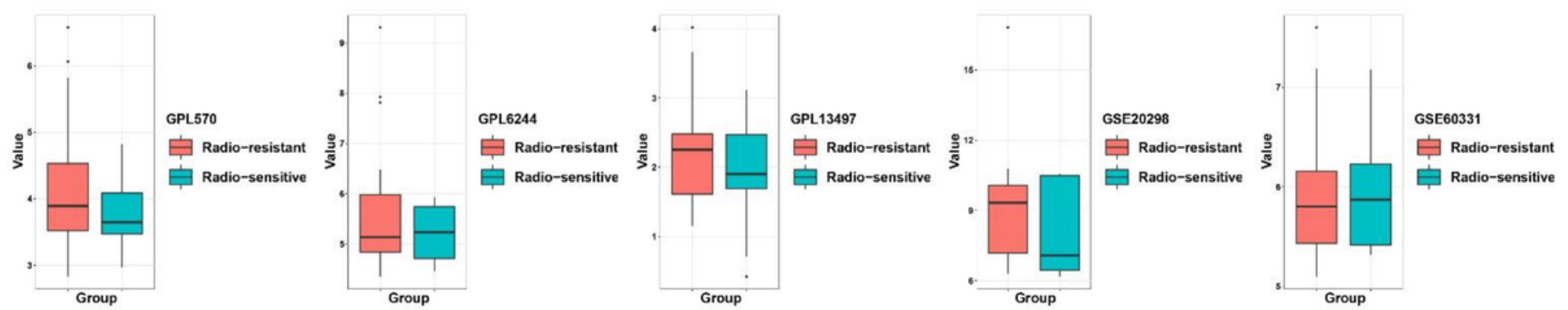

Figure 3

Boxplots showing TMPRSS3 expression in CRC radioresistant and radiosensitive tissues from different data sets.
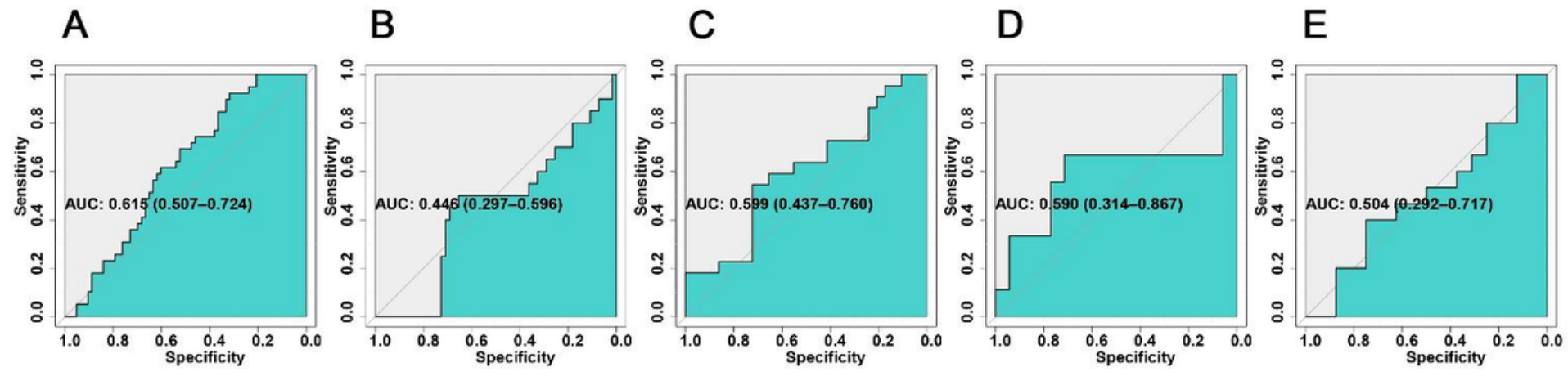

Figure 4

ROC curves for the classification model of TMPRSS3 in different CRC radioresistant and radiosensitive tissues. A. GPL570; B. GPL13497; C. GSE20298; D. GSE60331; E. GPL6244. 

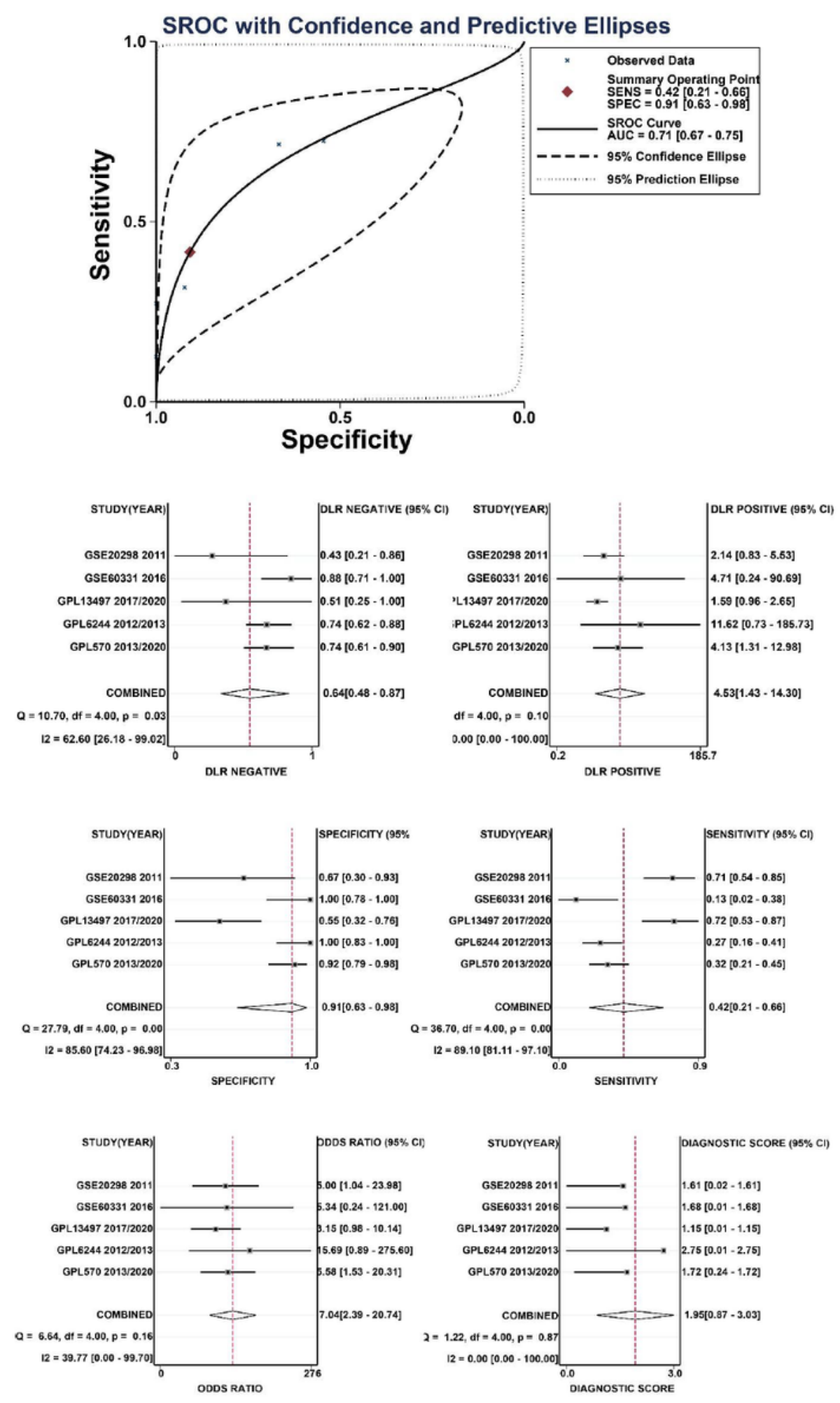

\section{Figure 5}

The SROC, DLR positive and negative, specificity, sensitivity, and diagnostic score of TMPRSS3 in all colorectal cancer radioresistant and radiosensitive tissues. The ability of TMPRSS3 to distinguish radioresistant from radiosensitive tissues has good specificity. 
Study

ID

GPL570

GPL13497

GSE20298

GSE60331

Overall (I-squared $=0.0 \%, p=0.758$ )
GPL6244

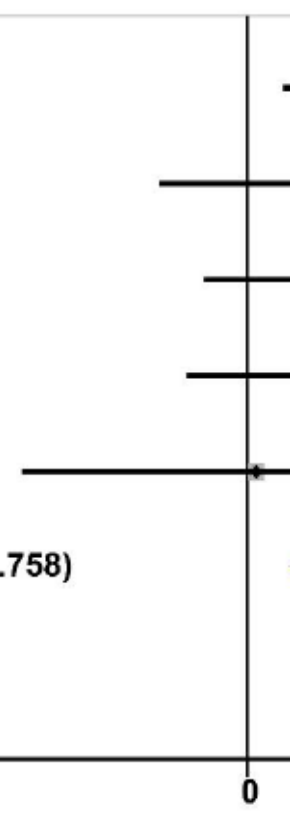

B Meta-analysis estimates, given named study is omitted Lower Cl Limit Estimate Upper Cl Limit

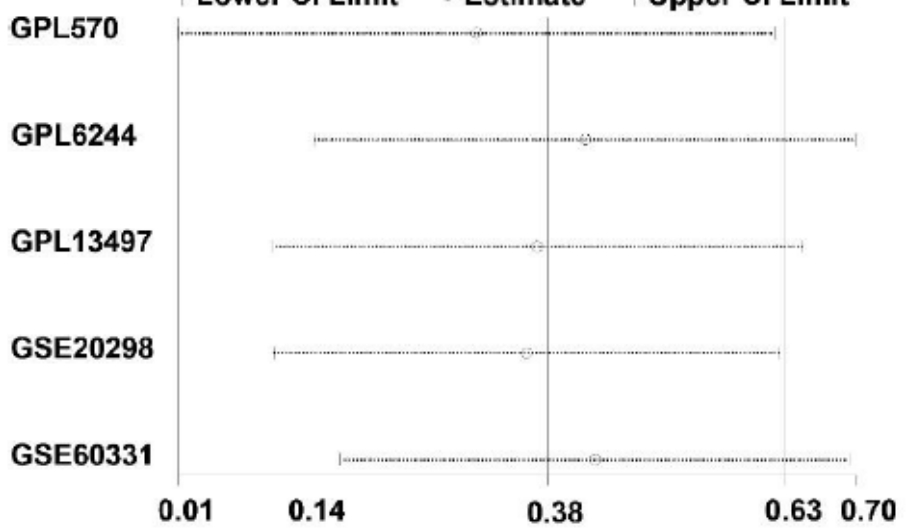

$\%$

Weight

$\operatorname{SMD}(95 \% \mathrm{Cl})$

$0.51(0.11,0.92) \quad 36.00$

$0.25(-0.26,0.76) \quad 22.47$

$0.43(-0.13,0.99) \quad 18.84$

$0.56(-0.18,1.30) \quad 10.75$

$0.03(-0.68,0.73) \quad 11.93$

$0.38(0.14,0.63) \quad 100.00$

C

Begg's funnel plot with pseudo $95 \%$ confidence limits

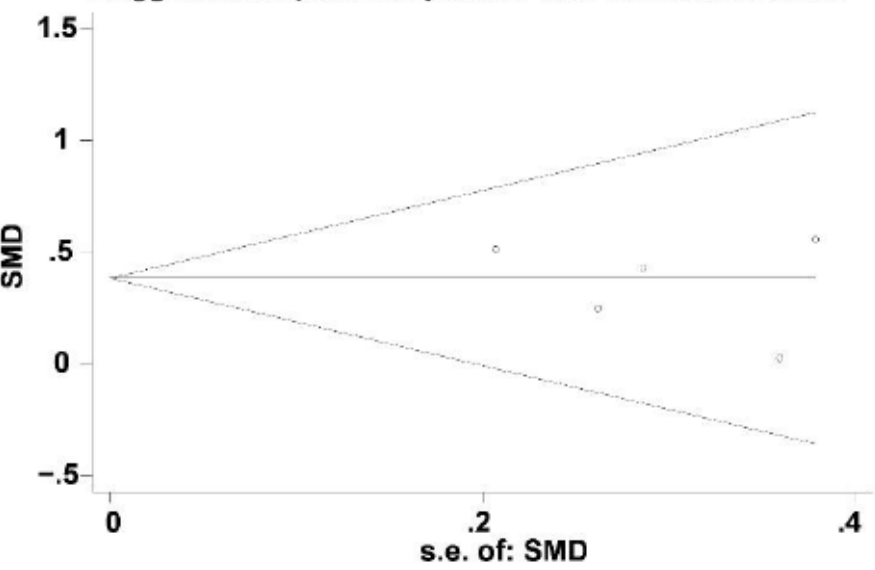

Figure 6

Meta-analysis results of TMPRSS3 expression in colorectal cancer radioresistance microarrays. A. Pooled Forest plots based on the expression of TMPRSS3 in all CRC radioresistant and radiosensitive data sets; B. Sensitivity analysis; C. Publication bias (Begg's Test). The above figures show that TMPRSS3 is highly expressed in $\mathrm{CRC}$ radioresistant tissues. 

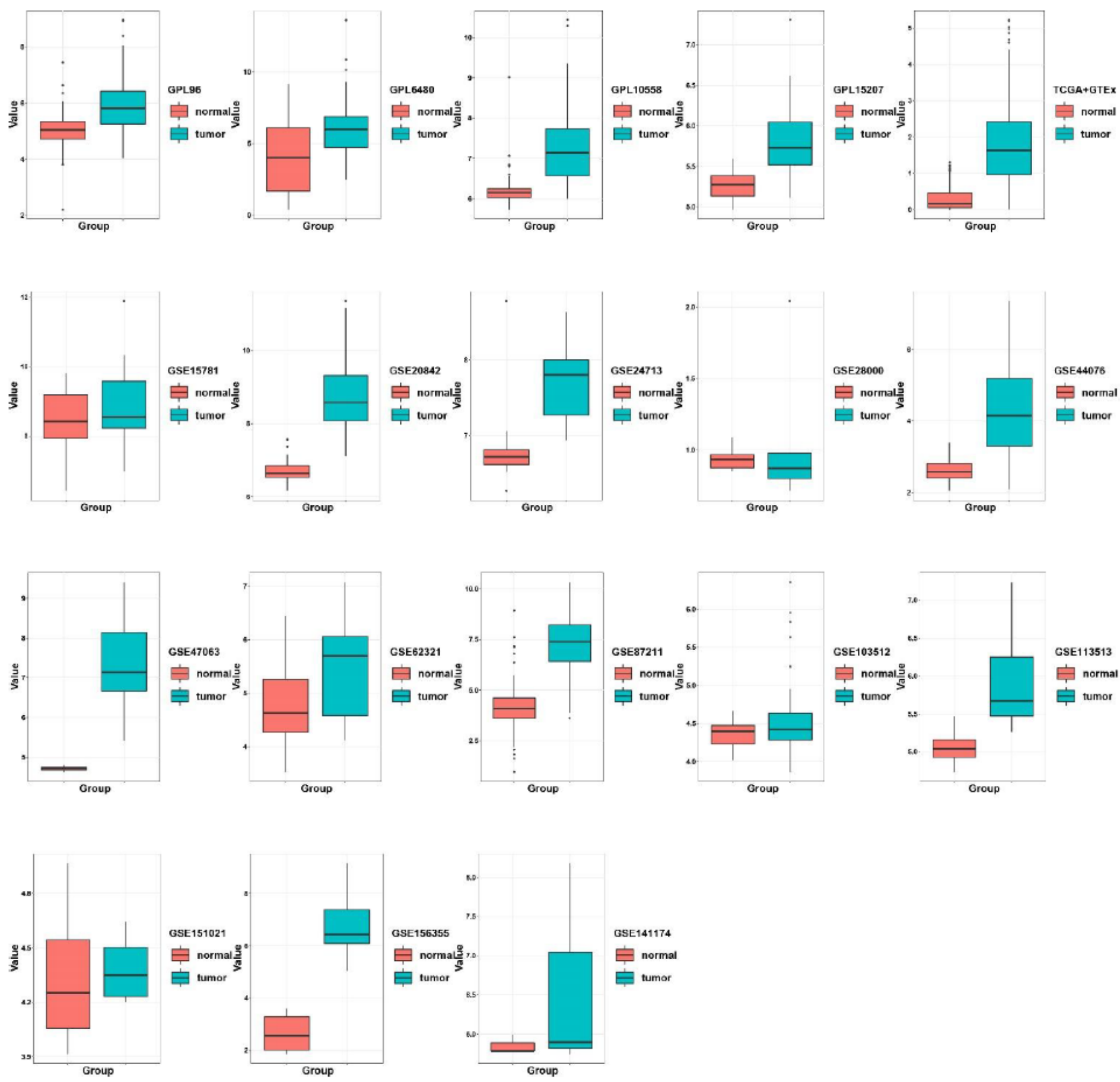

Figure 7

Box plots depicting the expression of TMPRSS3 in 26 data sets. The levels of expression are higher in cancer samples compared to adjacent tissues. 

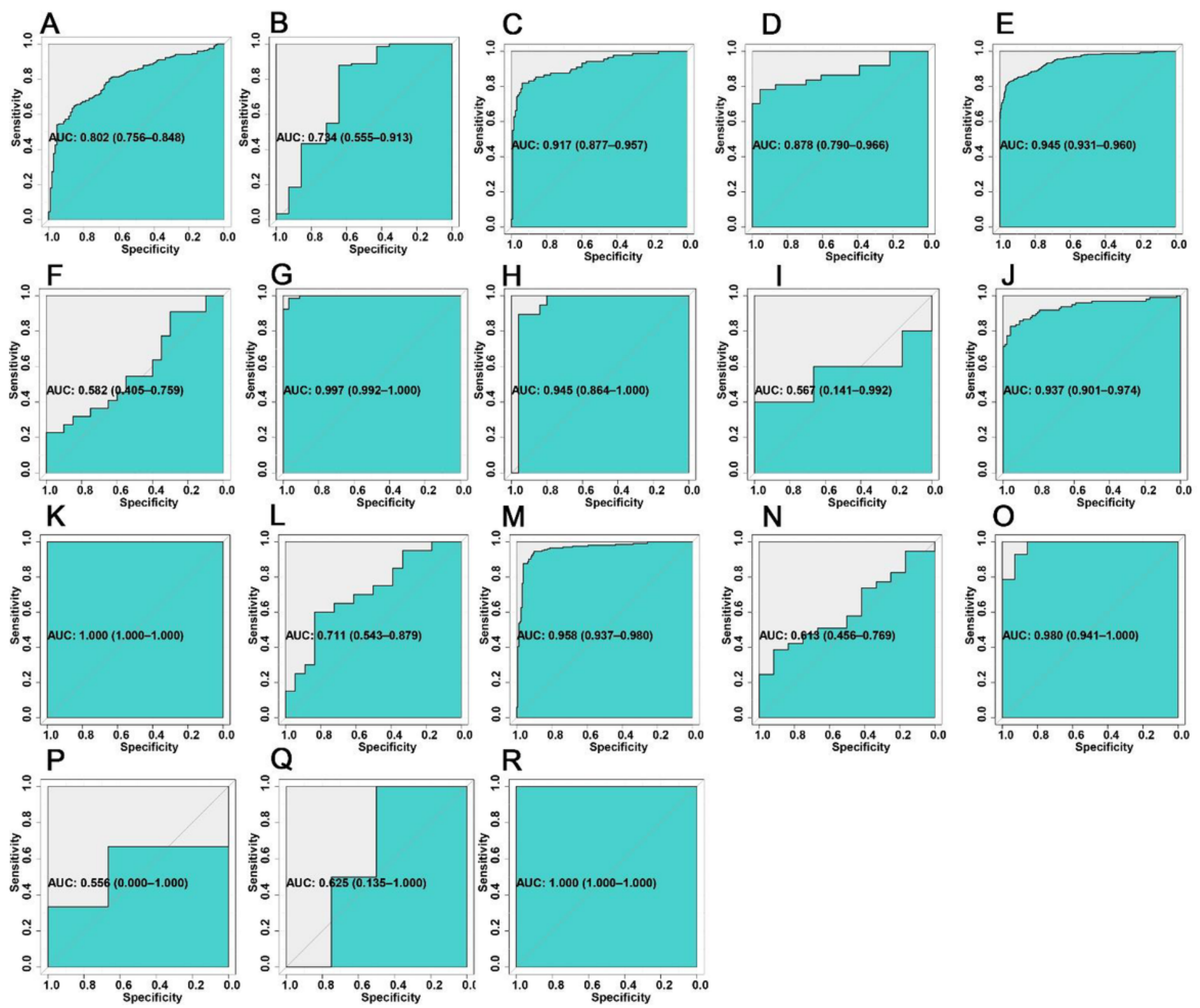

\section{Figure 8}

ROC curves of TMPRSS3 classification models in different colorectal cancer microarrays. AUC suggests that TMPRSS3 has a good ability to distinguish cancer tissues from paracancerous tissues. A. GPL96; B. GPL6480; C. GPL10558; D. GPL15207; E. TCGA+GTEx; F. GSE15781; G. GSE20842; H. GSE24713; I. GSE28000; J. GSE44076; K. GSE47063; L. GSE62321; M. GSE87211; N. GSE103512; O. GSE113513; P. GSE141174; Q. GSE151021; R. GSE156355. 
SROC with Confidence and Predictive Ellipses
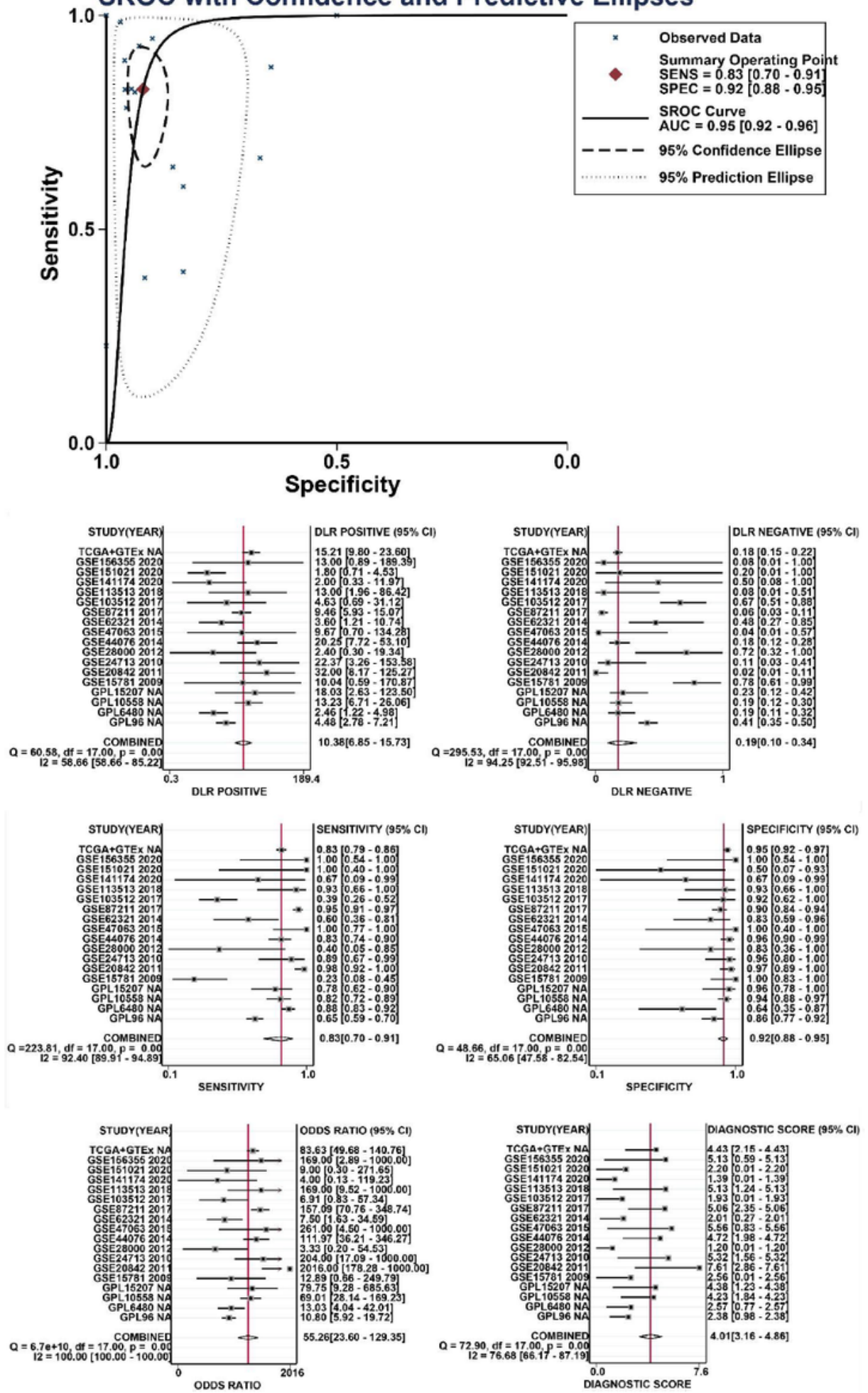

\section{Figure 9}

The SROC, DLR positive and negative, specificity, sensitivity, and diagnostic score of TMPRSS3 in all CRC microarrays. The results reveal that TMPRSS3 has good specificity for differentiating CRC from paracancerous tissues. The above plots show excellent specificity and sensitivity for the ability of TMPRSS3 to discriminate CRC tissues from paracancerous tissues. 
A

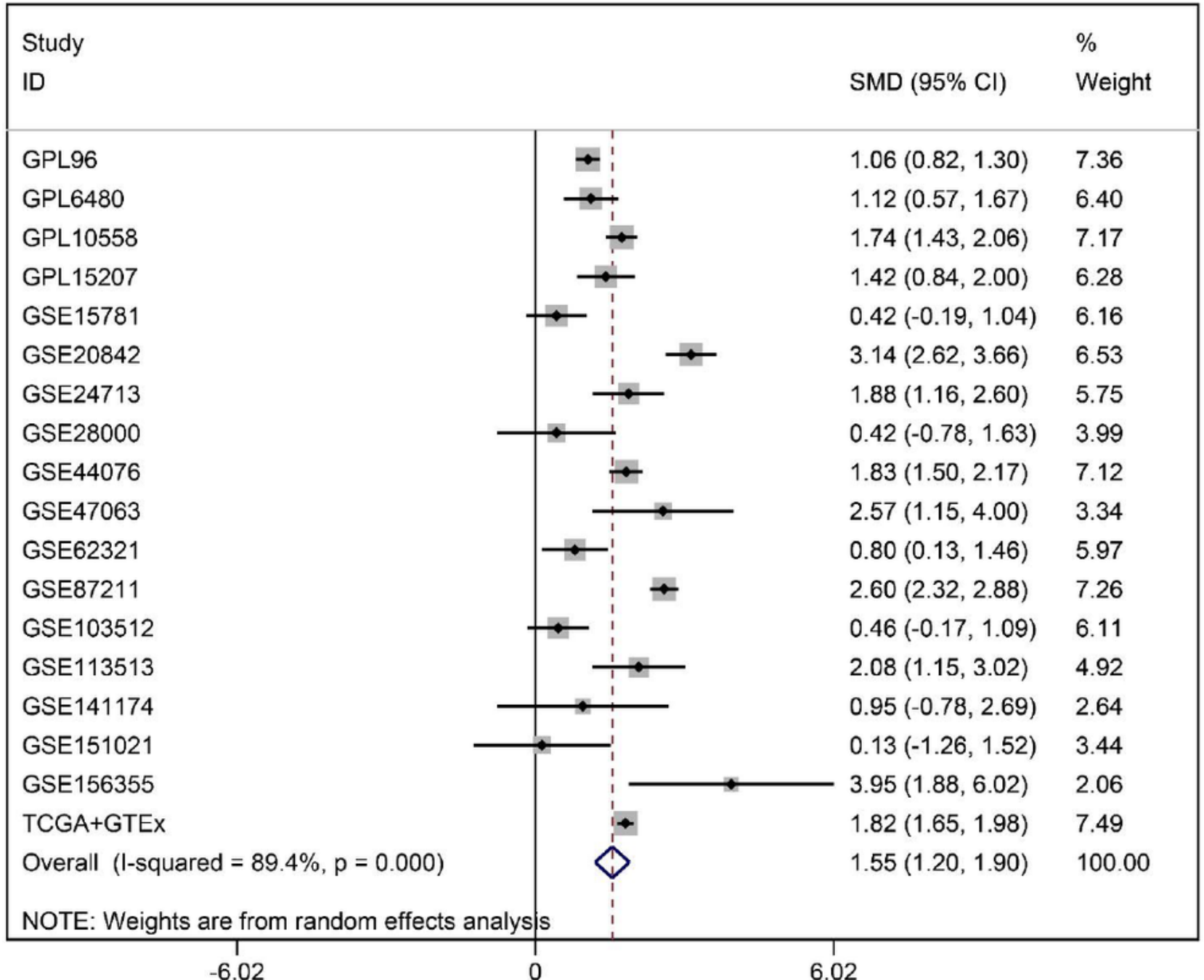

B $-6.02$

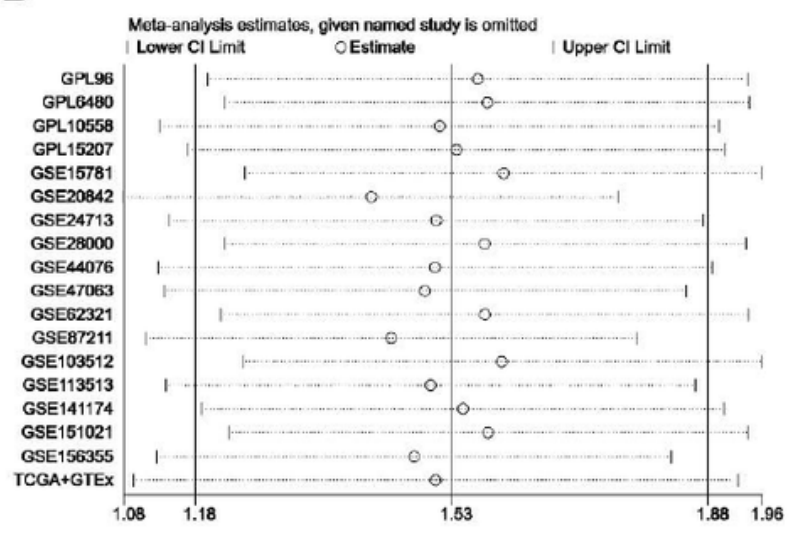

C

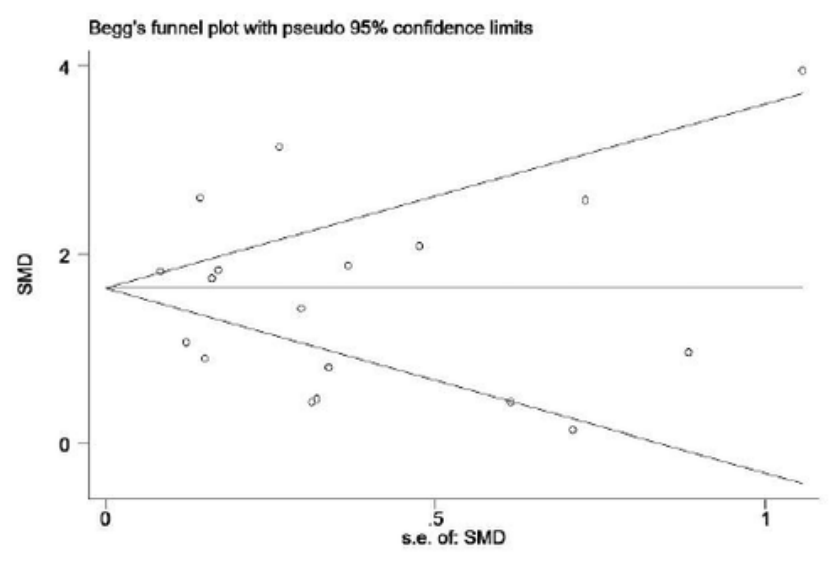

\section{Figure 10}

Meta-analysis results of TMPRSS3 expression in CRC. A. TMPRSS3 pooled forest plot based on all CRC microarrays; B. Sensitivity analysis; C. Publication bias (Begg's Test). 
A

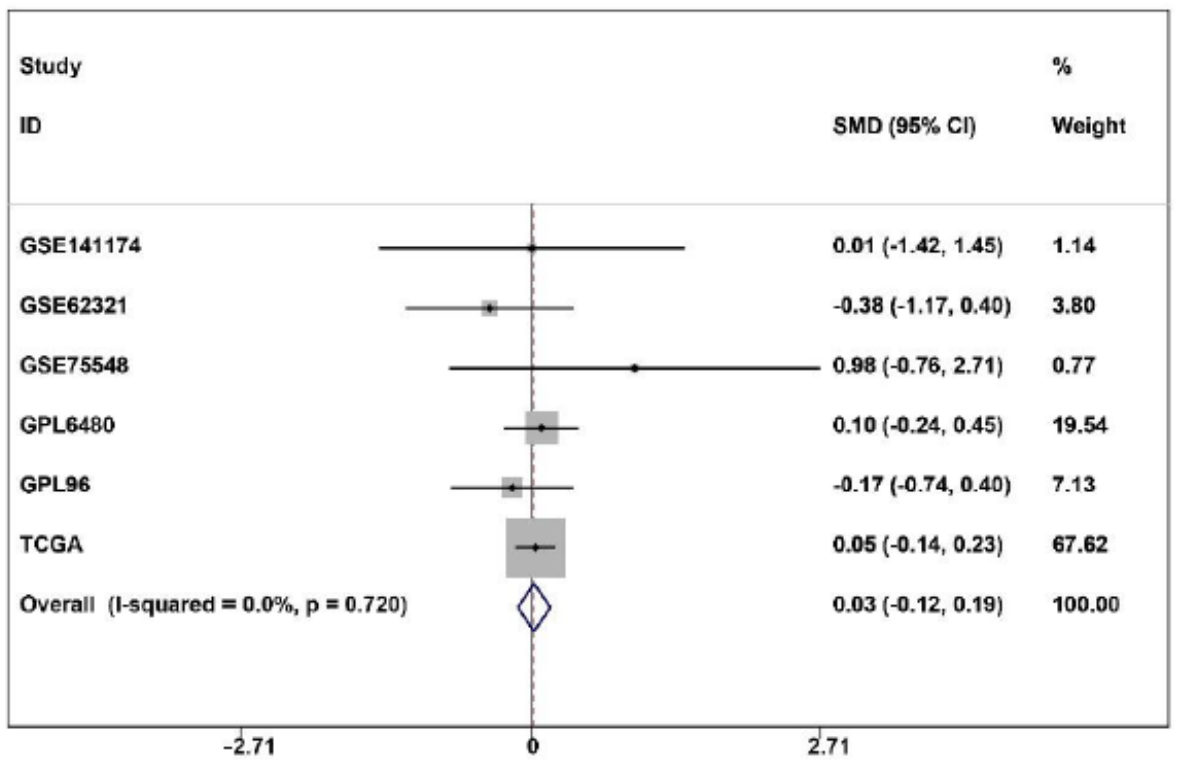

D

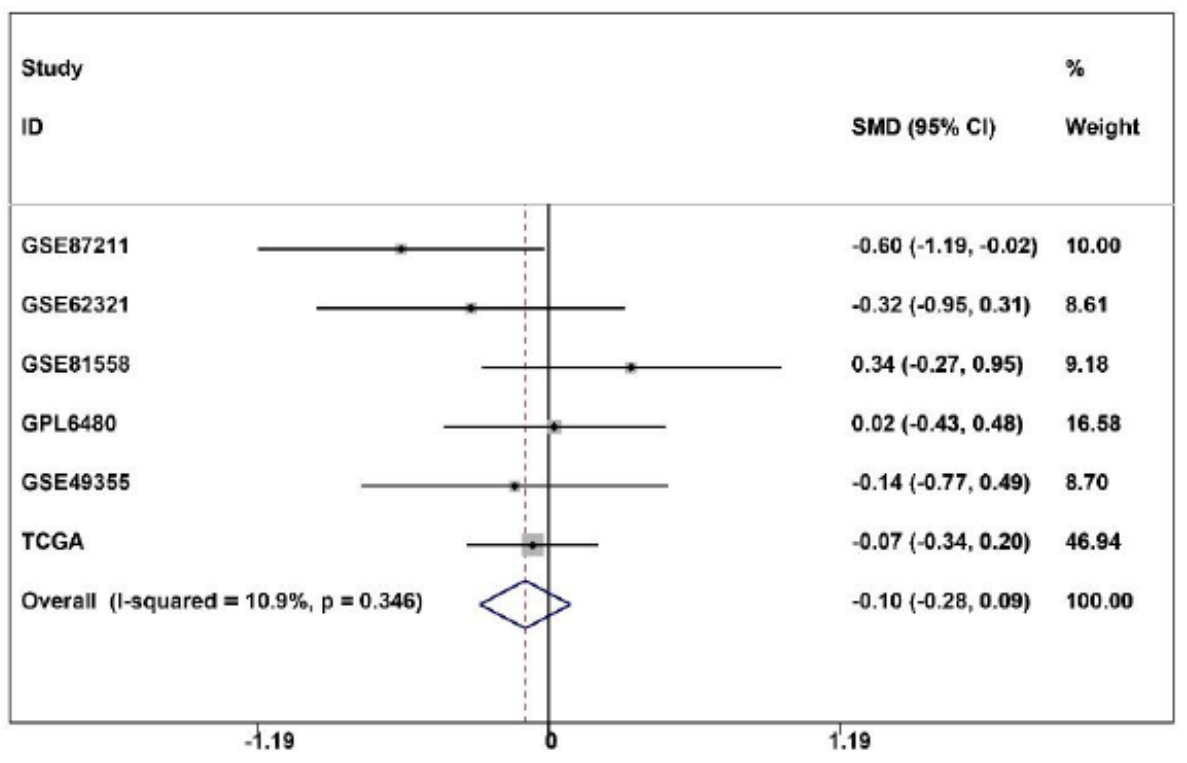

B
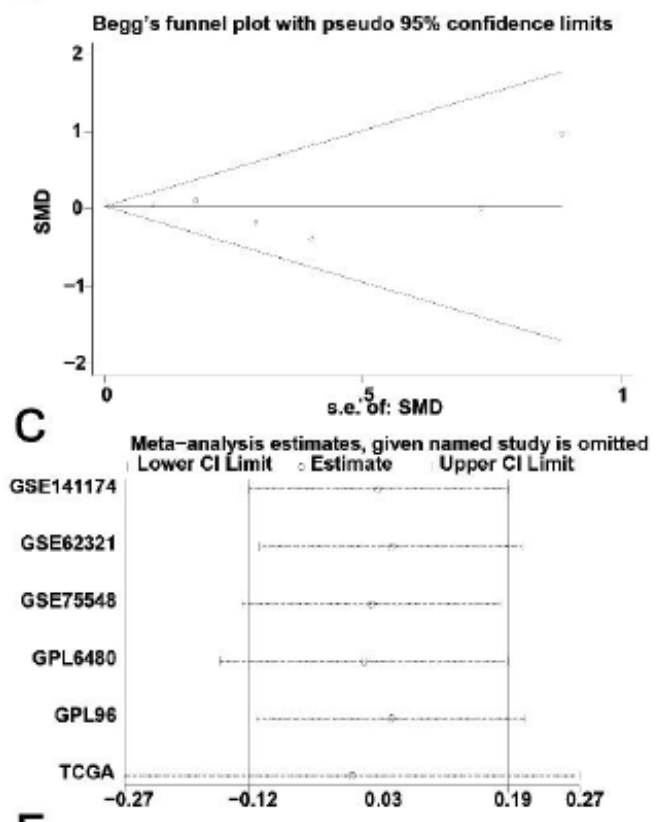

E

Begg's funnel plot with pseudo $95 \%$ confidence limits

.6
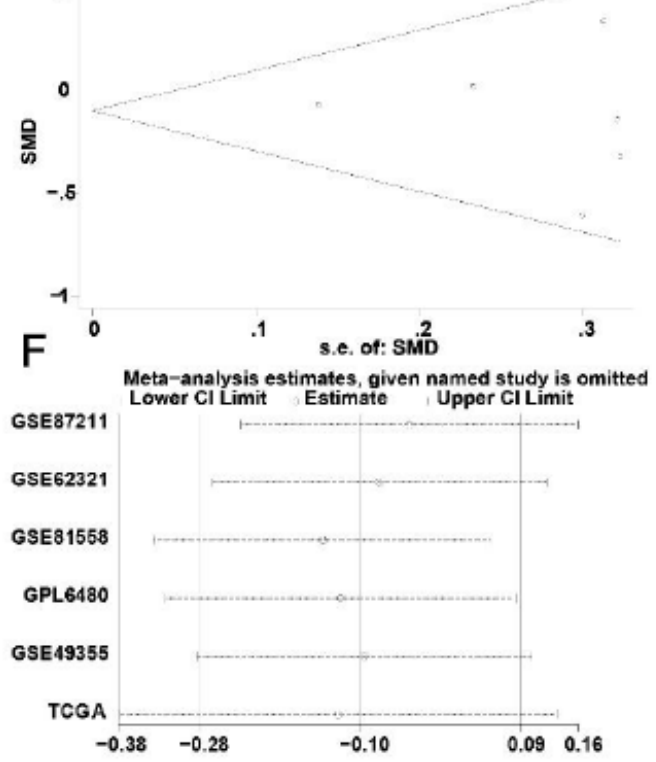

\section{Figure 11}

Meta-analysis of TMPRSS3 expression in lymph node metastasis and distant metastasis of CRC. A. Pooled forest plot of TMPRSS3 expression in lymph node metastasis and the results suggest no statistical significance; B. Sensitivity analysis of TMPRSS3 expression in lymph node metastasis; C. Data sets included in meta-analysis of TMPRSS3 expression in lymph node metastasis has no publication bias (Begg's Test). D. Pooled forest plot of TMPRSS3 expression in distant metastasis of CRC; the results suggest no statistical significance; E. Sensitivity analysis of TMPRSS3 expression in distant metastasis of CRC; F. Datasets included in the meta-analysis of TMPRSS3 in distant metastasis of CRC has no publication bias (Begg's Test). 

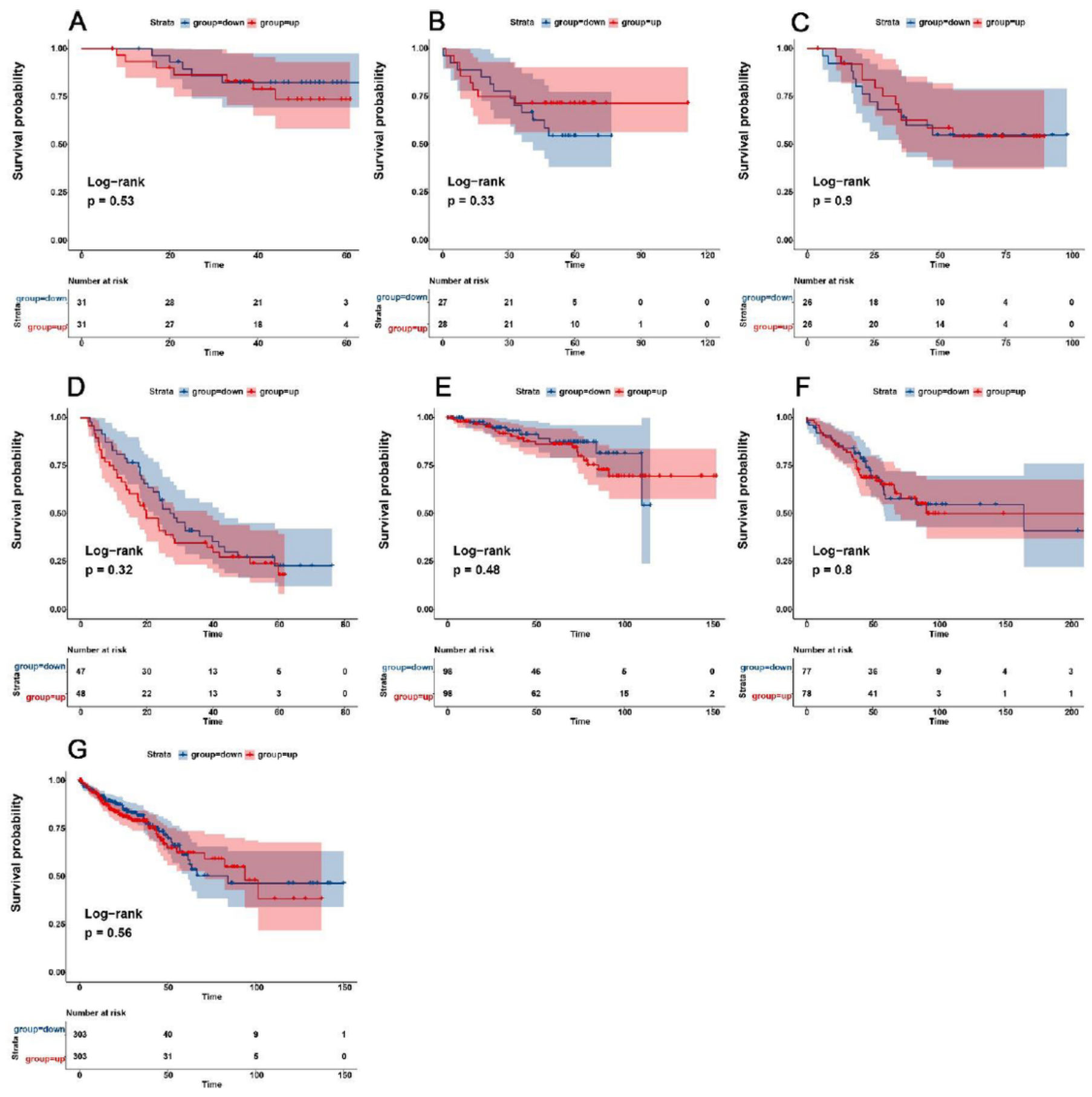

Figure 12

Kaplan-Meier curve of overall survival of TMPRSS3 in CRC. In the data set of large samples, it was seen that the OS of the low TMPSS3 expression group was significantly higher than that of the high expression group. A. GSE12945; B. GSE17537; C. GSE71187; D. GSE72970; E. GSE87211; F. GSE103479; G. TCGA. 

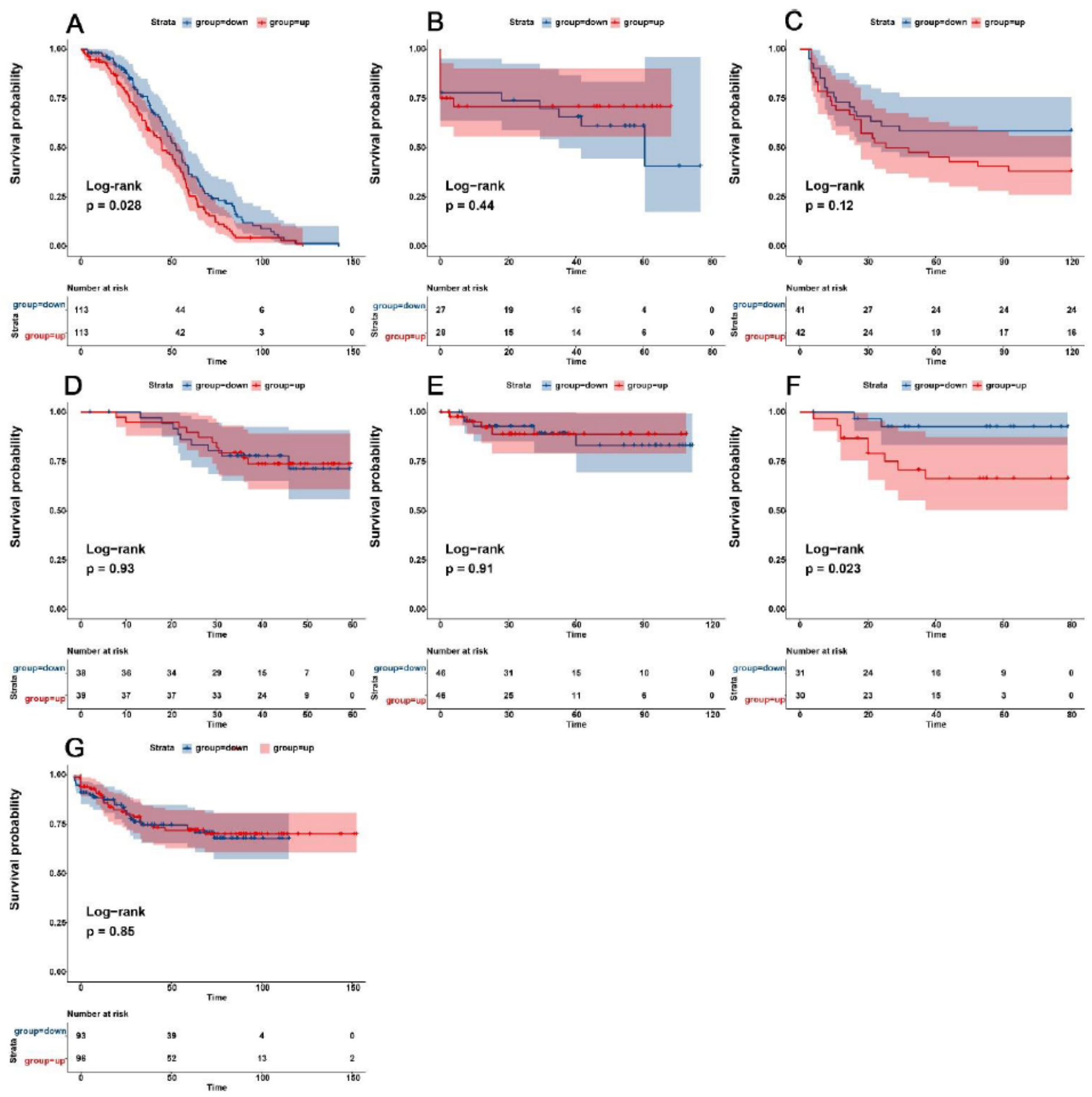

\section{Figure 13}

Kaplan-Meier curve of disease-free survival of TMPRSS3 in CRC. It is evident in the figure that the DFS of the low TMPRSS3 expression group is significantly better than that of the high expression group in the data set of large samples. A. GSE14333; B. GSE17537; C. GSE24549; D. GSE24550; E. GSE38832; F. GSE56699; G. GSE87211. 


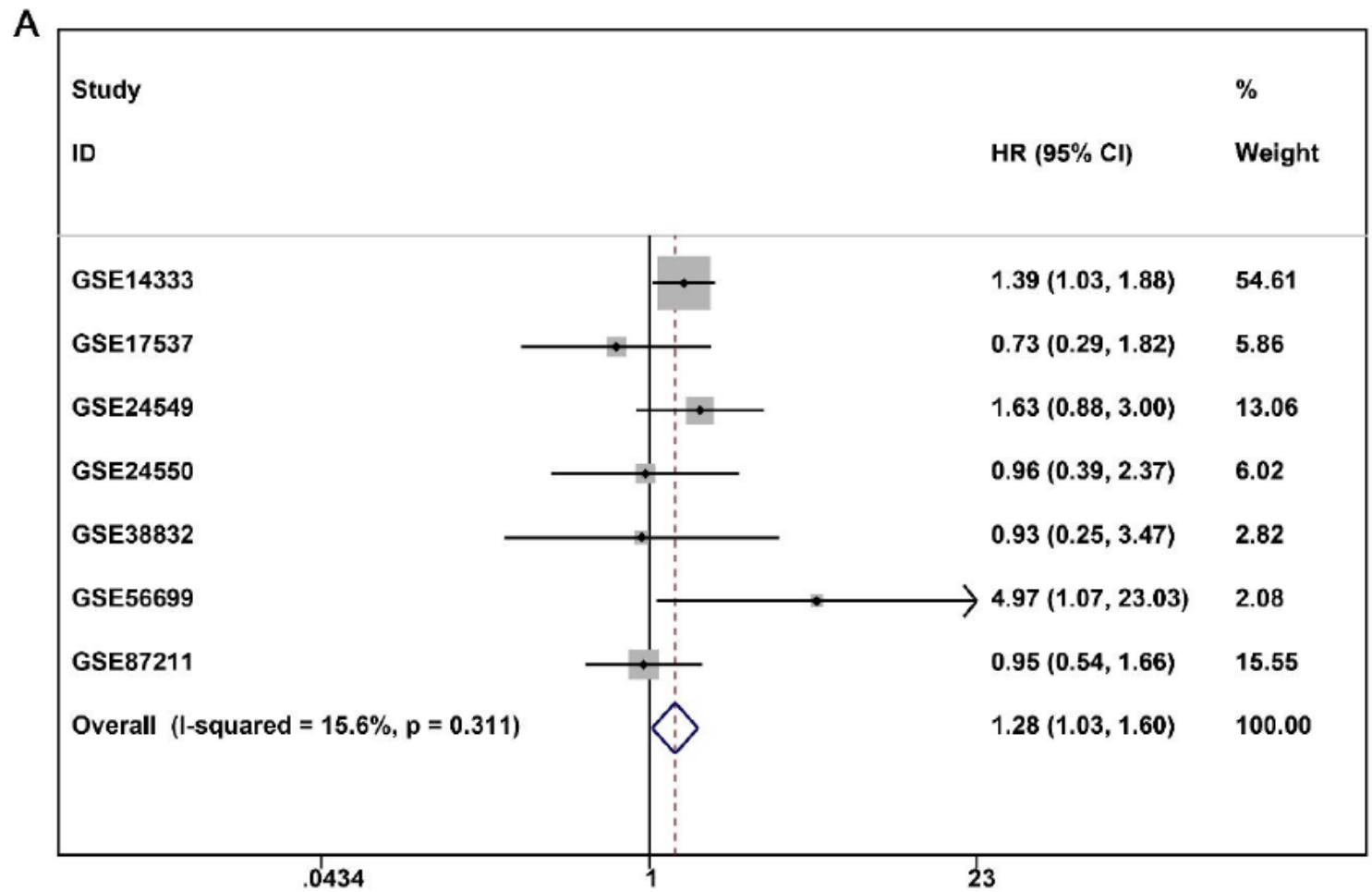

B

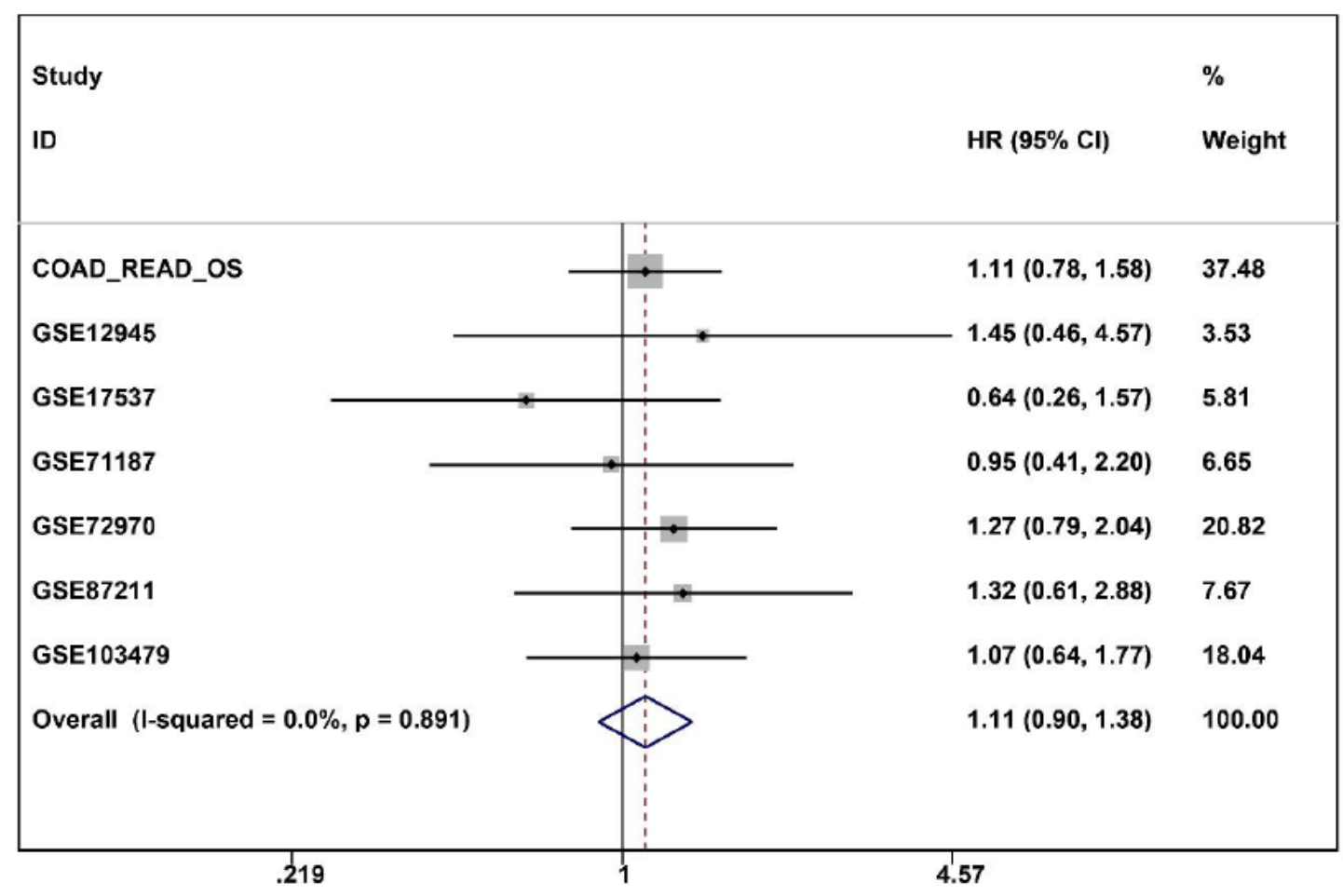

Figure 14

Pooled analysis of HRs obtained from COX analysis of OS and DFS. A. Pooled analysis of HR values for DFS data; B. Pooled analysis of HR values for OS data. 


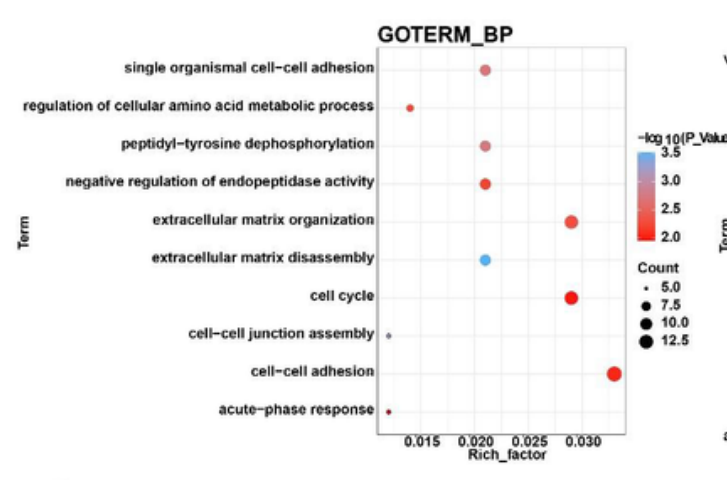

C

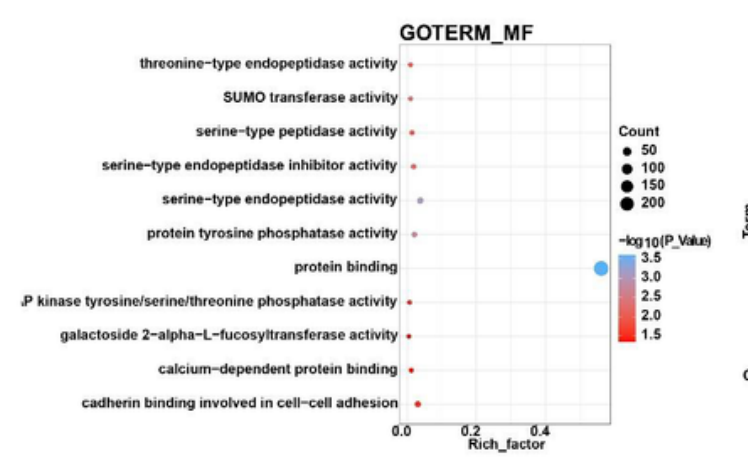

GOTERM_CC

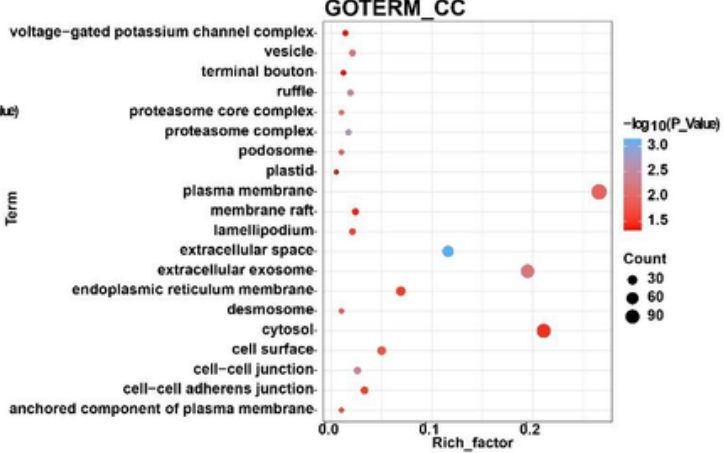

D

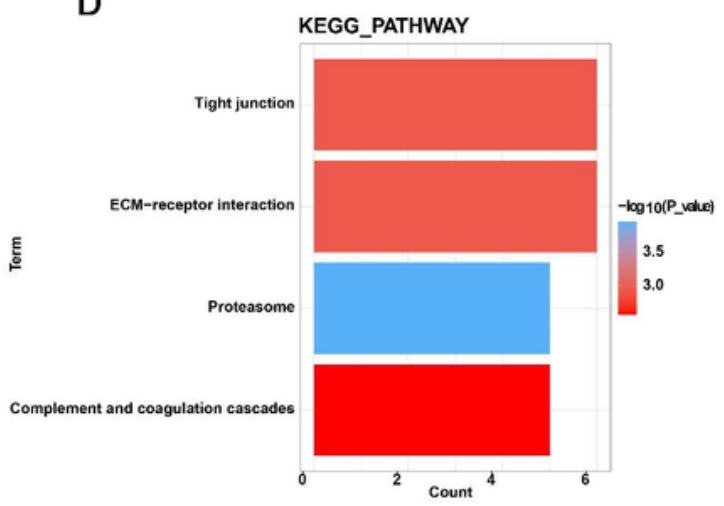

$\mathrm{E}$

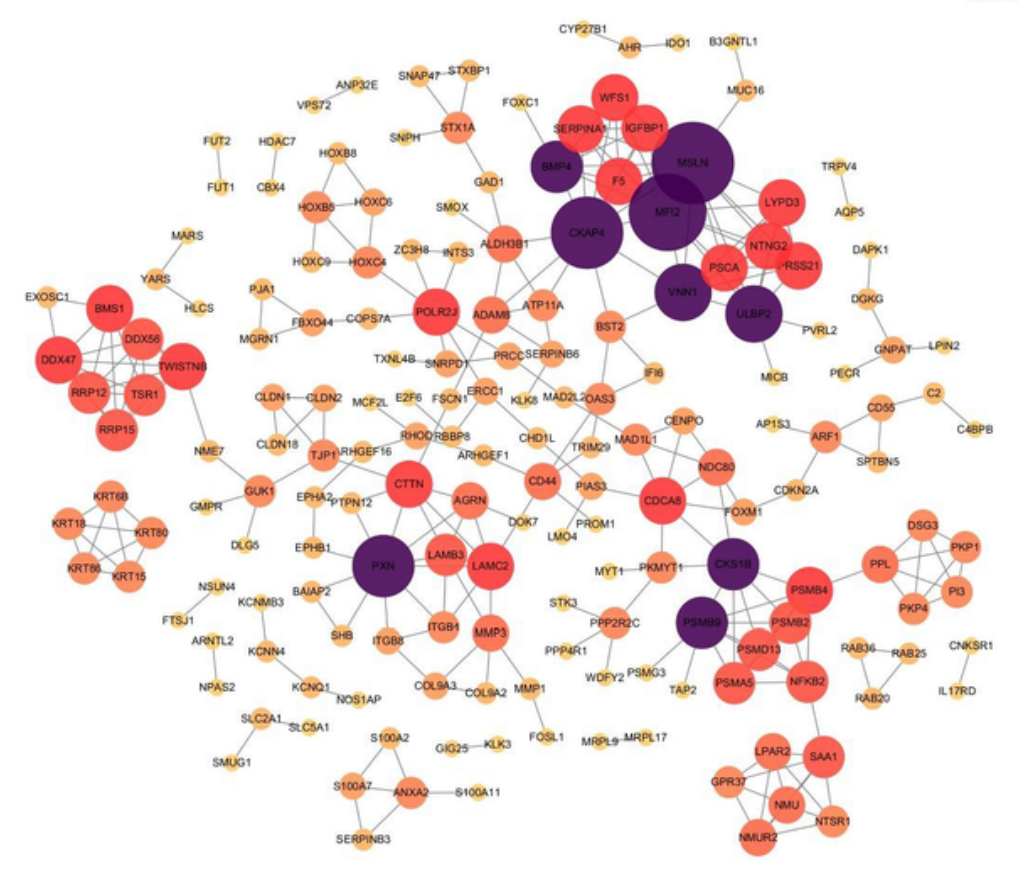

\section{Figure 15}

GO analysis, KEGG analysis, and construction of PPI network based on TMPRSS3 co-expressed genes. A. Biological process (BP); B. Cellular component (CC); C. Molecular function (MF) D. KEGG pathway; E. PPI network. 